NOTICE: this is the author's version of a work that was accepted for publication in Transport In Porous Media. Changes resulting from the publishing process, such as peer review, editing, corrections, structural formatting, and other quality control mechanisms may not be reflected in this document. Changes may have been made to this work since it was submitted for publication. A definitive version was subsequently published in Transport In Porous Media vol. 122 (1), pp. 57-76, March 2018 (https://doi.org/10.1007/s11242-017-0989-7). 


\title{
Upper bounds on the permeability of random porous media
}

\author{
François Bignonnet* \\ GeM, UMR CNRS 6183, Université de Nantes, Saint-Nazaire, \\ France
}

\begin{abstract}
Upper bounds on the permeability of random porous media are presented, which improve significantly on existing bounds. The derived bounds rely on a variational formulation of the upscaling problem from a viscous flow at the pore scale, described by Stokes equation, to a Darcy formulation at the macroscopic scale. A systematic strategy to derive upper bounds based on trial force fields is proposed. Earlier results based on uniform void or interface force fields are presented within this unified framework, together with a new proposal of surface force field and a combination of them. The obtained bounds feature detailed statistical information on the pore morphology, including two- and three-point correlation functions of the pore phase, the solid-fluid interface and its local orientation. The required spatial correlation functions are explicitly derived for the Boolean model of spheres, in which the solid phase is modelled as the union of penetrable spheres. Existing and new bounds are evaluated for this model and compared to full field simulations on representative volume elements. For the first time, bounds allow to retrieve the correct order of magnitude of permeability for a wide range of porosity and even improve on some estimates. However, none of the bounds reproduces the non-analytic behaviour of the permeability-porosity curve at low solid concentration.
\end{abstract}

Keywords-Random porous media ; Permeability ; Stokes flow ; Spatial correlation functions ; Homogenisation

\section{Introduction}

This work is part of a long-term effort that aims at finding reliable estimates or bounds on the permeability of porous media which rely solely on the description of the morphology of the material at the pore scale. For other physical properties of heterogeneous materials as the elastic modulii, the coefficient of diffusion or

*tel.: +33-2-40-17-81-89, e-mail: francois.bignonnet@univ-nantes.fr 
the electric conductivity, reliable upscaling tools are readily available such as Eshelby based schemes as well as the bounds of Hashin and Shtrikman (1962); Beran (1965); Ponte Castañeda and Willis (1995). For these latter physical properties, the governing equations have the same structure at the microscale and the macroscale. On the contrary, the present subject involves different descriptions of the flow depending on the scale: Stokes equations at the pore scale, but Darcy law at the macroscopic scale. Consequently, the strategies classically devised for the upscaling of elasticity or diffusion fail in the present case.

The problem of upscaling permeability from the description of the flow at the pore scale has been addressed by several means. Brinkman (1949) has empirically proposed an equation to bridge the Darcy and Stokes flow descriptions, upon which he has built a self-consistent approximation of the permeability of porous media made of spherical solid particles. Subsequent effective medium approximations have focused on the permeability of random arrays of spheres (Childress, 1972; Howells, 1974; Hinch, 1977; Kim and Russel, 1985) using a hierarchy of integro-differential equations. Wilkinson (1985) proposed an iterated dilute approximation based on Brinkman equation, instead of the early self-consistent approximation of Brinkman. An alternative strategy to estimate the permeability is to turn to particle-in-cell models (Happel, 1958; Kuwabara, 1959; Dassios et al, 1995; Boutin, 2000), which feature a simple morphological pattern comprising a single solid particle surrounded by a bounded cell of Newtonian fluid.

Additionally, following the pioneering work of Prager (1961), a series of rigorous upper bounds on the permeability of random media have been proposed, see Weissberg and Prager (1970); Doi (1976); Berryman (1983); Berryman and Milton (1985); Torquato and Beasley (1987); Rubinstein and Torquato (1989); Beasley and Torquato (1989); Torquato and Lu (1990); Given and Stell (1990); Willot et al (2016). The bounds are based on an energy definition of the permeability and choices of trial viscous stress fields.

As deplored by Given and Stell (1990), early authors had little reliable elements of comparison to assess the accuracy of the bounds. Following the development of efficient simulation tools (see e.g. Wiegmann, 2007; Bignonnet and Dormieux, 2014), full field simulation data on representative volume elements of random media data are now available for some random pore morphologies (Abdallah et al, 2015; Willot et al, 2016; Röding et al, 2017).

This paper introduces new upper bounds on the permeability of random media which improve on available ones. First, $\S 2$ provides background on statistical measures of random porous media: the spatial correlation functions. Then, $\S 3$ presents the problem for upscaling the permeability of a porous media at the macroscopic scale from Stokes flow at the microscopic scale. $\S 4$ introduces a variational framework to systematically derive upper bounds on the permeability, in which the spatial correlation function naturally arise from the choice of trial force fields. The evaluation of the bounds is provided in $\S 5$ for a simple model of granular porous media, the Boolean model of spheres. The accuracy of the new bounds is discussed through a comparison with dedicated numerical 
results and existing estimates.

\section{Spatial correlation functions of random porous media}

To start with, statistical descriptors of the microstructure that will arise in the bounds are presented. For a more general formalism, the reader is referred to Torquato $(1986,2002)$.

\section{$2.1 \quad$ Notations}

A Representative Volume Element (RVE) $\Omega$ of a porous medium comprises a fluid phase (or void phase) in the domain $\Omega_{f}$ and a solid phase in the domain $\Omega_{s}$, such that $\Omega_{f} \cup \Omega_{s}=\Omega$. Within the framework of random media, $\Omega_{f}$ and $\Omega_{s}$ are random sets. The indicator function $I_{v}$ of the void phase is defined as:

$$
I_{v}(\boldsymbol{z})= \begin{cases}1 & \text { if } \boldsymbol{z} \in \Omega_{f} \\ 0 & \text { otherwise }\end{cases}
$$

where $\boldsymbol{z}$ denotes the position vector in the RVE. In the following developments, the random field $I_{v}$ is assumed stationary and ergodic. The solid-fluid interface is denoted $\Gamma$. The indicator distribution of $\Gamma$ is the surface distribution $I_{s}$ defined such that for any field $f$ :

$$
\forall f, \quad \int_{\Omega} f(\boldsymbol{z}) I_{s}(\boldsymbol{z}) \mathrm{d} V_{z}=\int_{\Gamma} f(\boldsymbol{z}) \mathrm{d} S_{z}
$$

Some of the improved bounds on the permeability derived in $\S 4$ also involve information on the local orientation of the unit normal $\boldsymbol{n}$ to the solid-fluid interface $\Gamma$. Denoting by $\otimes$ the tensorial product, it will prove useful introduce the following tensorial surface distribution $\boldsymbol{I}_{o}$ such that:

$$
\forall f, \quad \int_{\Omega} f(\boldsymbol{z}) \boldsymbol{I}_{o}(\boldsymbol{z}) \mathrm{d} V_{z}=\int_{\Gamma} f(\boldsymbol{z}) \boldsymbol{n}(\boldsymbol{z}) \otimes \boldsymbol{n}(\boldsymbol{z}) \mathrm{d} S_{z}
$$

\subsection{One-point correlation functions}

The one-point correlation function of the void phase $F_{v}$ is the probability that a point $\boldsymbol{z}$ in the porous medium lies in the void phase:

$$
F_{v}=\left\langle I_{v}(\boldsymbol{z})\right\rangle=\phi
$$

where $\langle\bullet\rangle$ denotes the ensemble average over all possible realisations of the random set. The assumptions of ergodicity and stationarity of $I_{v}$ imply that $F_{v}$ is independent of the observation point $z ; F_{v}$ then corresponds to the porosity $\phi=\left|\Omega_{f}\right| /|\Omega|$ of a RVE of the porous medium. Similarly, the one-point 
correlation function of the solid-fluid interface $F_{s}$ corresponds to the specific surface area $s=|\Gamma| / \Omega \mid$ :

$$
F_{s}=\left\langle I_{s}(\boldsymbol{z})\right\rangle=s
$$

The function $F_{s}$ corresponds to the limiting case when $h \rightarrow 0$ of $1 / h$ times the probability that a point $\boldsymbol{z}$ lies within a distance less than $h / 2$ to the solid-fluid interface. Note that in case of an isotropic medium, $\left\langle\boldsymbol{I}_{o}(\boldsymbol{z})\right\rangle=\frac{s}{3} \mathbf{1}$ where $\mathbf{1}$ is the second order identity tensor.

\section{3 n-point correlation functions}

The following two-point correlation functions are classically defined (Doi, 1976; Torquato, 2002):

$$
\begin{aligned}
& F_{v v}(\boldsymbol{r})=\left\langle I_{v}(\boldsymbol{z}) I_{v}(\boldsymbol{z}+\boldsymbol{r})\right\rangle \\
& F_{s v}(\boldsymbol{r})=\left\langle I_{s}(\boldsymbol{z}) I_{v}(\boldsymbol{z}+\boldsymbol{r})\right\rangle=F_{v s}(-\boldsymbol{r}) \\
& F_{s s}(\boldsymbol{r})=\left\langle I_{s}(\boldsymbol{z}) I_{s}(\boldsymbol{z}+\boldsymbol{r})\right\rangle
\end{aligned}
$$

The two-point correlation function of the void phase $F_{v v}(\boldsymbol{r})$ - or covariogram of the random set $\Omega_{f}$ - is the probability that two points separated by a prescribed vector $\boldsymbol{r}$ both lie in the void phase. The function $F_{s v}(\boldsymbol{r})$ is the limiting case when $h \rightarrow 0$ of $1 / h$ times the probability that a point $\boldsymbol{z}$ lies within a distance less than $h / 2$ to the solid-fluid interface and $\boldsymbol{z}+\boldsymbol{r}$ lies in the fluid phase. $F_{s s}(\boldsymbol{r})$ is the limiting case when $h \rightarrow 0$ of $1 / h^{2}$ times the probability that two points $\boldsymbol{z}$ and $\boldsymbol{z}+\boldsymbol{r}$ both lie within a distance less than $h / 2$ to the solid-fluid interface.

The definition of $F_{s s}$ involves the product of two surface distributions, which results in a line distribution to be interpreted as follows. For two surfaces $S_{1}$ and $S_{2}$ of $\Omega$, whose unit normals are respectively denoted $\boldsymbol{n}_{1}$ and $\boldsymbol{n}_{2}$ and whose indicator (surface) distributions are denoted $I_{1}$ and $I_{2}$, the product $I_{1} I_{2}$ is a line distribution such that:

$$
\forall f, \quad \int_{\Omega} I_{1}(\boldsymbol{z}) I_{2}(\boldsymbol{z}) f(\boldsymbol{z}) \mathrm{d} V_{z}=\int_{S_{1} \cap S_{2}} f(\boldsymbol{z}) \frac{\mathrm{d} L_{z}}{\sqrt{1-\left(\boldsymbol{n}_{1} \cdot \boldsymbol{n}_{2}\right)^{2}}}
$$

where the right hand side is a line integral over the intersection of $S_{1}$ and $S_{2}$, which may diverge if the two surfaces are tangent. Accordingly, $\lim _{\|\boldsymbol{r}\| \rightarrow 0} F_{s s}(\boldsymbol{r})=$ $+\infty$.

In a similar fashion, new tensorial spatial correlation functions which convey a statistical information on the orientation of the unit normal $\boldsymbol{n}$ to the solid-fluid interface $\Gamma$ are defined:

$$
\begin{aligned}
& \boldsymbol{F}_{o v}(\boldsymbol{r})=\left\langle\boldsymbol{I}_{o}(\boldsymbol{z}) I_{v}(\boldsymbol{z}+\boldsymbol{r})\right\rangle=\boldsymbol{F}_{v o}(-\boldsymbol{r}) \\
& \boldsymbol{F}_{o s}(\boldsymbol{r})=\left\langle\boldsymbol{I}_{o}(\boldsymbol{z}) I_{s}(\boldsymbol{z}+\boldsymbol{r})\right\rangle=\boldsymbol{F}_{s o}(-\boldsymbol{r}) \\
& \boldsymbol{F}_{o o}(\boldsymbol{r})=\left\langle\boldsymbol{I}_{o}(\boldsymbol{z}) \cdot \boldsymbol{I}_{o}(\boldsymbol{z}+\boldsymbol{r})\right\rangle
\end{aligned}
$$

A straightforward generalisation of these notations will be used for threepoint correlation functions:

$$
F_{v i j}(\boldsymbol{r}, \boldsymbol{s})=\left\langle I_{v}(\boldsymbol{z}) I_{i}(\boldsymbol{z}+\boldsymbol{r}) I_{j}(\boldsymbol{z}+\boldsymbol{s})\right\rangle
$$


where the subscripts $i$ and $j$ are chosen among $\{v, s, o\}$ and the dot product is to be used between $\boldsymbol{I}_{o}$ and $\boldsymbol{I}_{o}$ whenever $i=j=o$. Online Resource $1^{1}$ provides explicit expressions of the above correlation functions for a simple model of random medium: the Boolean model of spheres.

\section{Permeability homogenisation problem}

\subsection{Direct definition in the framework of periodic homog- enization}

The description of flow through porous media driven by a pressure gradient depends on the scale of observation. At the microscopic scale - or scale of the pores - the flow is that of an incompressible Newtonian fluid within the pore space governed by Stokes equations:

$$
\begin{array}{lr}
\nabla \cdot \boldsymbol{\sigma}=0 & \left(\Omega_{f}\right) \\
\boldsymbol{\sigma}=-p \mathbf{1}+\mu \nabla^{s} \boldsymbol{v} & \left(\Omega_{f}\right) \\
\nabla \cdot \boldsymbol{v}=0 & \left(\Omega_{f}\right) \\
\boldsymbol{v}=\mathbf{0} & (\Gamma)
\end{array}
$$

where $\boldsymbol{v}$ denotes the fluid velocity, $p$ the pressure, $\boldsymbol{\sigma}$ the Cauchy stress tensor and $\mu$ the viscosity.

At the macroscopic scale - or scale of the structure - the flow is described by Darcy's law. Darcy's law linearly relates the filtration velocity $\boldsymbol{V}$, which is the average of $\boldsymbol{v}$ over a RVE, to the macroscopic pressure gradient $\boldsymbol{\nabla} \boldsymbol{P}$ via the permeability $\boldsymbol{K}$ :

$$
\begin{aligned}
& \nabla \cdot \boldsymbol{V}=0 \\
& \boldsymbol{V}=-\boldsymbol{K} \cdot \boldsymbol{\nabla} \boldsymbol{P}
\end{aligned}
$$

The upscaling of permeability from viscous flow through porous media has been first investigated by Prager (1961). Ene and Sanchez-Palencia (1975) later derived the permeability homogenisation problem from multiscale asymptotic expansions of (10) (see also Rubinstein and Torquato, 1989; Boutin, 2000; Auriault and Sanchez-Palencia, 1977; Auriault et al, 2005; Whitaker, 1986). Multiscale asymptotic expansion techniques rely on the scale separation principle: let $L$ and $\ell$ be the characteristic lengths of the macroscopic and microscopic scales respectively, then $\epsilon=\ell / L \ll 1$ is assumed. The fields, of the space variable $\boldsymbol{x}$, are decomposed as a series expansion of powers of $\epsilon$ and functions of two space variables: the fast variable $\boldsymbol{z}=\boldsymbol{x} / \ell$ and the slow variable $\boldsymbol{y}=\boldsymbol{x} / L$ (Auriault, 2002). For example, the stress field is decomposed as:

$$
\boldsymbol{\sigma}(\boldsymbol{x})=\boldsymbol{\sigma}_{0}(\boldsymbol{y}, \boldsymbol{z})+\epsilon \boldsymbol{\sigma}_{1}(\boldsymbol{y}, \boldsymbol{z})
$$

and similarly for $\boldsymbol{v}$ and $p$. Using scaling arguments, Ene and Sanchez-Palencia (1975); Boutin (2000) have shown that $\boldsymbol{\sigma}_{0}$ and $p_{0}$ depend only on the slow

\footnotetext{
${ }^{1}$ Appendix A of the present document.
} 
variable $\boldsymbol{y}$ and represent the macroscopic trend: a macroscopic pressure gradient $\nabla_{y} p_{0}(\boldsymbol{y})$ in the fluid phase and a macroscopic stress gradient $\nabla_{y} \boldsymbol{\sigma}_{0}(\boldsymbol{y})$ in the solid phase (in the opposite direction, to counterbalance the fluid pressure gradient).

In turn, $\boldsymbol{\sigma}_{1}$ and $p_{1}$ are microscopic stress and pressure fluctuations about the macroscopic trends $\boldsymbol{\sigma}_{0}$ and $p_{0}$. These fluctuations are driven by the macroscopic fluid pressure gradient $\nabla_{y} p_{0}(\boldsymbol{y})$ which appears as a constant for the fast variable $\boldsymbol{z}$ and will be denoted $\boldsymbol{\nabla} \boldsymbol{P}$. The driving force $\boldsymbol{\nabla} \boldsymbol{P}$ is balanced by viscous forces which arise from $\nabla_{z}^{s} \boldsymbol{v}_{0}$ :

$$
\begin{array}{lr}
\nabla_{z} \cdot \boldsymbol{\sigma}_{1}-\boldsymbol{\nabla} \boldsymbol{P}=0 & \left(\Omega_{f}\right) \\
\boldsymbol{\sigma}_{1}=-p_{1} \mathbf{1}+\mu \nabla_{z}^{s} \boldsymbol{v}_{0} & \left(\Omega_{f}\right) \\
\nabla_{z} \cdot \boldsymbol{v}_{0}=0 & \left(\Omega_{f}\right) \\
\boldsymbol{v}_{0}=\mathbf{0} & (\Gamma) \\
p_{1}, \boldsymbol{v}_{0} \text { periodic } & \left(\Omega_{f} \cap \partial \Omega\right)
\end{array}
$$

In the framework of periodic homogenisation, periodic boundary conditions on $\boldsymbol{v}_{0}$ and $p_{1}$ are considered to close the problem (13). Rubinstein and Torquato (1989) instead imposed vanishing velocity and pressure fluctuation conditions on $\partial \Omega$. The latter choice is however somehow inconsistent since, due to the incompressibility of the flow, it implies that the average of the velocity over the RVE and hence the permeability should also vanish (see also (15)).

The boundary value problem (13) constitutes the permeability upscaling problem, on which the present work will focus. To simplify the notations, the indices in $\boldsymbol{\sigma}_{1}, p_{1}, \boldsymbol{v}_{0}$ and $\nabla_{z}$ will be dropped in the remainder of the document and $\boldsymbol{z}$, scaling as a length, denotes the position vector in the RVE. Since the problem (13) is linear, the velocity field can be expressed as a linear function of the sole loading parameter $\boldsymbol{\nabla P}$ :

$$
\boldsymbol{v}(\boldsymbol{z})=-\boldsymbol{k}(\boldsymbol{z}) \cdot \nabla \boldsymbol{P}
$$

where the second order tensor $\boldsymbol{k}(\boldsymbol{z})$ is the so-called velocity concentration field. Volume averaging of the velocity yields the macroscopic filtration velocity $\boldsymbol{V}=$ $\bar{v}$ :

$$
\boldsymbol{V}=-\overline{\boldsymbol{k}} \cdot \boldsymbol{\nabla} \boldsymbol{P}
$$

where the velocity field $\boldsymbol{v}$ is extended to zero in the solid phase and $\mathbf{\boldsymbol { }}=$ $\frac{1}{|\Omega|} \int_{\Omega} \bullet \mathrm{d} V_{z}$ denotes the volume average of $\bullet$ over the RVE. Comparison of (15) to (11) indicates that the average of the velocity concentration field $\overline{\boldsymbol{k}}$ corresponds to the permeability tensor $\boldsymbol{K}$ of the porous medium. The aim of the present work is to derive rigorous upper bounds on $\boldsymbol{K}$.

\subsection{Variational formulation in the framework of periodic homogenization}

The construction of bounds on the permeability relies on a minimum energy definition of the permeability. It will prove convenient to express it for fields 
defined on the whole RVE $\Omega$ instead of the sole fluid phase. To do so, the velocity field is extended to zero within the solid phase ; the set $\mathcal{K}$ of kinematically admissible velocity fields is then:

$$
\mathcal{K}=\left\{\boldsymbol{v} \mid \begin{array}{lr}
\begin{array}{l}
\text { continuous, } \nabla \cdot \boldsymbol{v}=0 \\
\text { periodic }
\end{array} & (\Omega) \\
(\partial \Omega)
\end{array}\right\}
$$

The stress fluctuation field has also to be extended to the solid phase. Just as the stress fluctuation in the fluid sees the macroscopic pressure gradient $\boldsymbol{\nabla} \boldsymbol{P}=\nabla_{y} p_{0}(\boldsymbol{y})$ as a body force within the pore space, the stress fluctuation in the solid sees the macroscopic stress divergence $\nabla_{y} \cdot \boldsymbol{\sigma}_{0}(\boldsymbol{y})$ as a body force within the solid phase (Boutin, 2000). Hence the stress fluctuation is subjected to a body force field $\boldsymbol{f}$ defined as follows. The restriction of $\boldsymbol{f}$ to $\Omega_{f}$ is $\boldsymbol{f}_{\left.\right|_{\Omega_{f}}}=-\boldsymbol{\nabla} \boldsymbol{P}$. Since the stress in the solid phase is indeterminate, the choice of $\boldsymbol{f}$ on $\Omega_{s}$ is arbitrary provided that the overall equilibrium of the RVE is ensured. Hence, the volume average of $\boldsymbol{f}$ over $\Omega$ must vanish to ensure that the stress fluctuation field is self-balanced. The set of admissible force fields $f$ is thus:

$$
\mathcal{F}(\boldsymbol{\nabla} \boldsymbol{P})=\left\{\boldsymbol{f} \mid \boldsymbol{f}(\boldsymbol{z})=-\boldsymbol{\nabla} \boldsymbol{P} \text { if } \boldsymbol{z} \in \Omega_{f}, \bar{f}=\mathbf{0}\right\},
$$

This could also be seen as the fact that the divergence of the macroscopic stress in the solid phase counterbalances the gradient of the macroscopic pressure in the fluid phase. To sum up, the set $\mathcal{S}(\boldsymbol{\nabla} \boldsymbol{P})$ of stress fluctuation fields statically admissible with the macroscopic pressure gradient $\boldsymbol{\nabla} \boldsymbol{P}$ is defined as:

$$
\mathcal{S}(\boldsymbol{\nabla} \boldsymbol{P})=\left\{\begin{array}{ll}
\boldsymbol{\sigma} & \begin{array}{l}
\boldsymbol{\sigma} \cdot \boldsymbol{n} \text { anti-periodic } \\
\exists \boldsymbol{f} \in \mathcal{F}(\boldsymbol{\nabla} \boldsymbol{P}), \nabla \cdot \boldsymbol{\sigma}+\boldsymbol{f}(\boldsymbol{z})=0
\end{array}
\end{array}\right\}
$$

The condition $\overline{\boldsymbol{f}}=0$ has generally been omitted by previous investigators seeking bounds on the permeability. As will be seen later in $\S 4.4$, it provides a physical insight to choices of trial fields. With these notations, the permeability homogenisation problem (10) amounts to:

$$
\text { find } \boldsymbol{v} \in \mathcal{K}, \boldsymbol{\sigma} \in \mathcal{S}(\boldsymbol{\nabla P}) \quad \text { such that } \quad \mathbb{K}: \boldsymbol{\sigma}=2 \mu \nabla^{s} \boldsymbol{v} \quad\left(\Omega_{f}\right)
$$

where $\mathbb{K}$ is the fourth order projection tensor on the space of second order deviatoric tensors operating as $\mathbb{K}: \boldsymbol{\sigma}=\boldsymbol{\sigma}-\operatorname{tr}(\boldsymbol{\sigma}) \mathbf{1} / 3$.

The variational formulation of the permeability homogenisation problem (19) is based on Hill's lemma:

$$
\forall \boldsymbol{v} \in \mathcal{K}, \forall \boldsymbol{\sigma} \in \mathcal{S}(\boldsymbol{\nabla P}) \quad \overline{I_{v} \boldsymbol{\sigma}: \nabla^{s} \boldsymbol{v}}=-\boldsymbol{\nabla} \boldsymbol{P} \cdot \overline{\boldsymbol{v}}
$$

which is a direct consequence of the divergence theorem and the definitions of $\mathcal{K}$ and $\mathcal{S}(\boldsymbol{\nabla} \boldsymbol{P})$. Let $(\boldsymbol{v}, \boldsymbol{\sigma})$ be the couple solution to problem (19). Then any stress field $\boldsymbol{\sigma}^{\prime} \in \mathcal{S}(\boldsymbol{\nabla} \boldsymbol{P})$ decomposes in $\boldsymbol{\sigma}^{\prime}=\boldsymbol{\sigma}+\delta \boldsymbol{\sigma}$ where $\delta \boldsymbol{\sigma}=\boldsymbol{\sigma}^{\prime}-\boldsymbol{\sigma} \in \mathcal{S}(0)$ by linearity. Multiple application of (20) yields $\forall \boldsymbol{\sigma}^{\prime} \in \mathcal{S}(\boldsymbol{\nabla} \boldsymbol{P})$ :

$$
\overline{\frac{I_{v}}{2 \mu} \boldsymbol{\sigma}^{\prime}: \mathbb{K}: \boldsymbol{\sigma}^{\prime}}=\underbrace{\overline{I_{\boldsymbol{v}} \boldsymbol{\sigma}: \nabla^{s} \boldsymbol{v}}}_{=\boldsymbol{\nabla} \boldsymbol{P} \cdot \boldsymbol{K} \cdot \boldsymbol{\nabla} \boldsymbol{P}}+\frac{1}{2 \mu} \underbrace{\overline{I_{b} \delta \boldsymbol{\sigma}: \mathbb{K}: \delta \boldsymbol{\sigma}}}_{\geq 0}+2 \overline{\underbrace{\overline{I_{b} \delta \boldsymbol{\sigma}: \nabla^{s} \boldsymbol{v}}}_{=0}}
$$


The energy definition of the permeability $\boldsymbol{K}$ then follows:

$$
\boldsymbol{\nabla} \boldsymbol{P} \cdot \boldsymbol{K} \cdot \boldsymbol{\nabla} \boldsymbol{P}=\inf _{\boldsymbol{\sigma}^{\prime} \in \mathcal{S}(\nabla \boldsymbol{P})} \overline{\frac{I_{v}}{2 \mu} \boldsymbol{\sigma}^{\prime}: \mathbb{K}: \boldsymbol{\sigma}^{\prime}}
$$

\subsection{Stochastic definition of the permeability in the frame- work of statistically homogeneous ergodic media}

In the framework of statistically homogeneous periodic media, the permeability definitions presented in the previous sections hold for any realisation $\omega$ of a RVE of a rigid porous media. Further, due to the formal analogy between periodic media and statistically homogeneous ergodic media (Sab, 1994), the stochastic definition of the permeability proposed by Rubinstein and Torquato (1989) actually amounts to replacing the volume averages over the RVE in (15), $(20),(21)$ and (22) by ensemble averages $\langle\bullet\rangle$ over all possible realisations of the random media at an arbitrary point $\boldsymbol{z}$. In further developments, these two frameworks will be interchanged whenever convenient.

\section{Upper bounds on the permeability}

\subsection{A general method to derive upper bounds based on force fields}

In this section, the variational principle (22) is applied to trial fields constructed using the Green operators. The idea is closely related to the polarisation techniques which are used in linear elasticity and lead to the Hashin and Shtrikman (1962) and Beran (1965) bounds. To construct trial fields for the initial heterogeneous homogenisation problem (19), an auxiliary problem is considered in which all the domain $\Omega$ is filled with a uniform Newtonian fluid of viscosity $\mu$. The heterogeneity and the no-slip condition are dropped and will have to be accounted for by an appropriate choice of the force field applied to the homogeneous fluid. The boundary conditions on $\partial \Omega$ are kept similar to those on the initial problem. The auxiliary problem is thus defined as follows:

$$
\begin{array}{ll}
\nabla \cdot \boldsymbol{\sigma}^{\prime}+\boldsymbol{f}=0 & (\Omega) \\
\boldsymbol{\sigma}^{\prime}=-p^{\prime} \mathbf{1}+2 \mu \nabla^{s} \boldsymbol{v}^{\prime} & (\Omega) \\
\nabla \cdot \boldsymbol{v}^{\prime}=0 & (\Omega) \\
\boldsymbol{v}^{\prime} \text { and } p^{\prime} \text { periodic } & (\partial \Omega)
\end{array}
$$

where $\boldsymbol{f}$ is an arbitrary volume force field whose choice will have to be optimised. The force field $\boldsymbol{f}$ which allows to retrieve the solution to the initial problem (19) is the Lagrange multiplier of the no-slip boundary condition. The motivation to consider such auxiliary problem is that the velocity field solution to the problem (23) is formally known as:

$$
\boldsymbol{v}^{\prime}(\boldsymbol{z})=\int_{\Omega} \boldsymbol{G}(\boldsymbol{z}-\boldsymbol{y}) \cdot \boldsymbol{f}(\boldsymbol{y}) \mathrm{d} V_{y}
$$


where $\boldsymbol{G}$ is by definition the Green function of the incompressible fluid of viscosity $\mu$ on the domain $\Omega$ with periodic boundary conditions. Consequently, the strain rate field solution to the auxiliary problem (23) is given by:

$$
\nabla^{s} \boldsymbol{v}^{\prime}(\boldsymbol{z})=\int_{\Omega} \mathcal{G}(\boldsymbol{z}-\boldsymbol{y}) \cdot \boldsymbol{f}(\boldsymbol{y}) \mathrm{d} V_{y}
$$

where $\mathcal{G}_{i j k}=\frac{1}{2}\left(\boldsymbol{G}_{i k, j}+\boldsymbol{G}_{j k, i}\right)$ will be referred to as the third order Green operator.

Provided that the volume force field $\boldsymbol{f}$ is chosen in the set $\mathcal{F}(\boldsymbol{\nabla P})$ of admissible force fields (17), the trial stress field $\boldsymbol{\sigma}^{\prime}$ solution to the auxiliary problem (23) is by construction an admissible field for the initial problem (19), i.e. $\boldsymbol{\sigma}^{\prime} \in \mathcal{S}(\boldsymbol{\nabla} \boldsymbol{P})$. The variational principle (22) then implies $\forall \boldsymbol{f} \in \mathcal{F}(\boldsymbol{\nabla} \boldsymbol{P})$ :

$\boldsymbol{\nabla} \boldsymbol{P} \cdot \boldsymbol{K} \cdot \boldsymbol{\nabla} \boldsymbol{P} \leq \frac{2 \mu}{|\Omega|} \iiint_{\Omega} I_{v}(\boldsymbol{z})(\mathcal{G}(\boldsymbol{z}-\boldsymbol{x}) \cdot \boldsymbol{f}(\boldsymbol{x})):(\mathcal{G}(\boldsymbol{z}-\boldsymbol{y}) \cdot \boldsymbol{f}(\boldsymbol{y})) \mathrm{d} V_{x} \mathrm{~d} V_{y} \mathrm{~d} V_{z}$

A weaker but simpler bound can be obtained by successive use of $I_{v}(\boldsymbol{z}) \leq 1$, Green's formula to the couple $\left(\boldsymbol{\sigma}^{\prime}, \nabla^{s} \boldsymbol{v}^{\prime}\right)$ and (24):

$$
\boldsymbol{\nabla} \boldsymbol{P} \cdot \boldsymbol{K} \cdot \boldsymbol{\nabla} \boldsymbol{P} \leq \frac{1}{|\Omega|} \iint_{\Omega} \boldsymbol{f}(\boldsymbol{z}) \cdot \boldsymbol{G}(\boldsymbol{z}-\boldsymbol{y}) \cdot \boldsymbol{f}(\boldsymbol{y}) \mathrm{d} V_{y} \mathrm{~d} V_{z}
$$

Equality can be met in (26) and (27): consider the solution $\boldsymbol{\sigma}$ to the flow problem in the fluid phase (unique), and extend it to zero velocity and stress in the solid phase. This solution can be readily retrieved from the Green operator applied to the force field $\boldsymbol{f}^{\text {opt }}$ defined as $-\boldsymbol{\nabla} \boldsymbol{P}$ in $\Omega_{f}, 0$ in $\Omega_{s}$ and a surface distribution of forces $\boldsymbol{\sigma} \cdot \boldsymbol{n}$ on $\Gamma$.

By linearity of the problems at hand, suitable force fields must be chosen linear with respect to the macroscopic pressure gradient $\boldsymbol{\nabla} \boldsymbol{P}$. Let us introduce the field of force concentration tensor $\boldsymbol{A}$ such that $\boldsymbol{f}(\boldsymbol{z})=\boldsymbol{A}(\boldsymbol{z}) \cdot \boldsymbol{\nabla} \boldsymbol{P}$, then (27) and (26) respectively become:

$$
\begin{aligned}
& \boldsymbol{K} \preceq \frac{1}{|\Omega|} \iint_{\Omega} \boldsymbol{A}^{T}(\boldsymbol{z}) \cdot \boldsymbol{G}(\boldsymbol{z}-\boldsymbol{y}) \cdot \boldsymbol{A}(\boldsymbol{y}) \mathrm{d} V_{y} \mathrm{~d} V_{z} \\
& \boldsymbol{K} \preceq \frac{2 \mu}{|\Omega|} \iiint_{\Omega} I_{v}(\boldsymbol{z}) \boldsymbol{A}^{T}(\boldsymbol{x}) \cdot \mathcal{G}^{T}(\boldsymbol{z}-\boldsymbol{x}): \mathcal{G}(\boldsymbol{z}-\boldsymbol{y}) \cdot \boldsymbol{A}(\boldsymbol{y}) \mathrm{d} V_{x} \mathrm{~d} V_{y} \mathrm{~d} V_{z}
\end{aligned}
$$

where $\preceq$ stands for the inequality in the sense of quadratic forms and the convention for the transposition of a third order tensor is $\mathcal{G}_{i j k}^{T}=\mathcal{G}_{j k i}$. Since the considered Green operators are translation independent, the change of variables $\boldsymbol{r}=\boldsymbol{z}-\boldsymbol{y}$ and $\boldsymbol{s}=\boldsymbol{z}-\boldsymbol{x}$ allow to re-express the bounds (28) as:

$$
\begin{aligned}
& \boldsymbol{K} \preceq \int \boldsymbol{G}(\boldsymbol{r}): \mathbb{A}^{2 \mathrm{pts}}(\boldsymbol{r}) \mathrm{d} V_{r} \\
& \boldsymbol{K} \preceq 2 \mu \iint\left(\mathcal{G}^{T}(\boldsymbol{r}): \mathcal{G}(\boldsymbol{s})\right): \mathbb{A}^{3 \mathrm{pts}}(\boldsymbol{r}, \boldsymbol{s}) \mathrm{d} V_{r} \mathrm{~d} V_{s}
\end{aligned}
$$


where $\mathbb{A}^{2 \mathrm{pts}}(\boldsymbol{r})$ and $\mathbb{A}^{3 \mathrm{pts}}(\boldsymbol{r}, \boldsymbol{s})$ are fourth-order tensor carrying information on two- and three-point correlations respectively, defined by:

$$
\begin{aligned}
\mathbb{A}_{i j k l}^{2 \mathrm{pts}}(\boldsymbol{r}) & =\left\langle\boldsymbol{A}_{j k}(\boldsymbol{z}) \boldsymbol{A}_{i l}(\boldsymbol{z}+\boldsymbol{r})\right\rangle \\
\mathbb{A}_{i j k l}^{3 \mathrm{pts}}(\boldsymbol{r}, \boldsymbol{s}) & =\left\langle I_{v}(\boldsymbol{z}) \boldsymbol{A}_{j k}(\boldsymbol{z}+\boldsymbol{r}) \boldsymbol{A}_{i l}(\boldsymbol{z}+\boldsymbol{s})\right\rangle
\end{aligned}
$$

In (30), the volume average over the RVE in the periodic homogenisation framework is replaced by an ensemble average over all realisations of the random media as stated in $\S 3.3$ in the framework of random media. The bounds (29) hold for any force localisation tensor $\boldsymbol{A}$ such that $\boldsymbol{A} \cdot \boldsymbol{\nabla} \boldsymbol{P} \in \mathcal{F}(\boldsymbol{\nabla} \boldsymbol{P})$. The bounds are assessed in $\S 4.4$ for specific choices of the force fields, for which $\mathbb{A}^{2 \mathrm{pts}}(\boldsymbol{r}, \boldsymbol{s})$ and $\mathbb{A}^{3 \mathrm{pts}}(\boldsymbol{r}, \boldsymbol{s})$ are combinations of the two- and three-point correlation functions introduced in $\S 2$.

\subsection{Approximation of the Green operators}

For the bounds to be fully explicit, a classical simplifying approximation is conceded: provided that there is no long-range order in the porous medium and that the size of the domain $\Omega$ is much larger than the caracteristic size of correlation of the random medium, the infinite-body Green operators will be substituted for the finite-body Green operators defined on $\Omega$. The infinite-body Green function $G^{\infty}$ (or Stokeslet) and the third-order Green operator $\mathcal{G}^{\infty}$ of the incompressible Newtonian fluid are translation invariant and explicitly known as functions of $\boldsymbol{r}=\boldsymbol{z}-\boldsymbol{y}$ :

$$
\begin{aligned}
\boldsymbol{G}^{\infty}(\boldsymbol{r}) & =\frac{\mathbf{1}+\boldsymbol{e}_{r} \otimes \boldsymbol{e}_{r}}{8 \pi \mu r} \\
\mathcal{G}^{\infty}(\boldsymbol{r}) & =\frac{\mathbf{1} \otimes \boldsymbol{e}_{r}-3 \boldsymbol{e}_{r} \otimes \boldsymbol{e}_{r} \otimes \boldsymbol{e}_{r}}{8 \pi \mu r^{2}} \quad \text { with } \quad r=|\boldsymbol{r}| ; \boldsymbol{e}_{r}=\frac{\boldsymbol{r}}{r}
\end{aligned}
$$

The simplifying approximation of substituting the infinite-body Green operators for those of the finite-body is classically performed in elasticity homogenisation for the fourth-order Green operator while assessing the bounds of Hashin and Shtrikman (1962); it has been justified by Willis (1977) in the framework of kinematically uniform boundary conditions. In the latter framework, the infinite-body fourth-order Green operator has to be applied to the fluctuation of the polarisation field around its average to avoid an unwanted behaviour with uniform polarisation fields. Similarly in the present setting, the Green function and the third order Green operator of the infinite body have to be applied to the fluctuation of the force field around its average, namely to $\boldsymbol{f}-\overline{\boldsymbol{f}}$. Since the overall equilibrium imposes here to consider trial force fields satisfying $\bar{f}=0$, the infinite-body Green operators will directly be applied to $f$.

\subsection{Simplification of the bounds for isotropic media}

Let us now focus on isotropic media ; then $\boldsymbol{K}=K \mathbf{1}$ is an isotropic tensor. Furthermore, provided that the choice of the force concentration field $\boldsymbol{A}$ does not 
introduce a preferential orientation, the quantity $a^{3 \mathrm{pts}}(r, s, u)\left(\operatorname{resp} \cdot a^{2 \mathrm{pts}}(r)\right)$ defined below only depends on $r=|\boldsymbol{r}|, s=|\boldsymbol{s}|$ and $u=\boldsymbol{e}_{r} \cdot \boldsymbol{e}_{s}$ (resp. on $r$ ):

$$
\begin{aligned}
a^{2 \mathrm{pts}}(r) & =\left(\mathbf{1}+\boldsymbol{e}_{r} \otimes \boldsymbol{e}_{r}\right): \mathbb{A}^{2 \mathrm{pts}}(\boldsymbol{r}): \mathbf{1} \\
a^{3 \mathrm{pts}}(r, s, u) & =\boldsymbol{e}_{r} \otimes \boldsymbol{e}_{s}: \mathbb{A}^{3 \mathrm{pts}}(\boldsymbol{r}, \boldsymbol{s}): \mathbf{1}
\end{aligned}
$$

where $\boldsymbol{e}_{r}=\boldsymbol{r} / r$ and $\boldsymbol{e}_{s}=\boldsymbol{s} / r$. Using these definitions and (31), the two- and three-point bounds (29) simplify after integration over appropriate angles to:

$$
\begin{aligned}
& K \leq \frac{1}{6 \mu} \int_{r=0}^{\infty} r a^{2 \mathrm{pts}}(r) \mathrm{d} r \\
& K \leq \frac{1}{4 \mu} \int_{r=0}^{\infty} \int_{s=0}^{\infty} \int_{u=-1}^{1}\left(3 u^{2}-1\right) a^{3 \mathrm{pts}}(r, s, u) \mathrm{d} r \mathrm{~d} s \mathrm{~d} u
\end{aligned}
$$

where use has been made of:

$$
\mathcal{G}^{\infty^{T}}(\boldsymbol{r}): \mathcal{G}^{\infty}(\boldsymbol{s})=\frac{3 \boldsymbol{e}_{r} \otimes \boldsymbol{e}_{s}\left(3\left(\boldsymbol{e}_{r} \cdot \boldsymbol{e}_{s}\right)^{2}-1\right)}{(8 \pi \mu r s)^{2}}
$$

\subsection{Trial force fields}

This section introduces several explicit choices of admissible trial force fields involved in the above bounds. First, two trial fields classically used in the literature - uniform volume and surface force fields - are presented in the light of the framework of $\S 4.1$. Second, a new force field is proposed to improve the statistical description of the solid-fluid interface. Finally, sharper bounds are derived by combining all these force fields in the variational formulation of $\S 4.1$.

The simplest choice of admissible force field complying with (17) is a phasewise uniform force field, which will be referred to as the void force field, and whose concentration tensor is:

$$
\boldsymbol{A}_{v}=\frac{\phi-I_{v}}{1-\phi} \mathbf{1}
$$

In the case of an isotropic media, the application of the two- and three-point bounds (33) to the void force field (35) reveal the two- and three-point correlation functions of the void phase:

$$
\begin{aligned}
& K \leq K_{v v, 2 \mathrm{pts}}=\frac{2}{3 \mu(1-\phi)^{2}} \int_{r=0}^{\infty} r\left(F_{v v}(r)-\phi^{2}\right) \mathrm{d} r \\
& K \leq K_{v v, 3 \mathrm{pts}}=\frac{1}{4 \mu(1-\phi)^{2}} \int_{r=0}^{\infty} \int_{s=0}^{\infty} \int_{u=-1}^{1}\left(3 u^{2}-1\right) u F_{v v v}(r, s, u) \mathrm{d} r \mathrm{~d} s \mathrm{~d} u
\end{aligned}
$$

The void bound (35) corresponds to the proposal of Prager (1961). In the original work of Prager (1961), a coefficient 9/10 had been found instead of $2 / 3$, 
as pointed out and corrected by Berryman and Milton (1985). It can be shown that the bounds of Berryman and Milton (1985) are equivalent to the Prager one's provided a third order Green operator for compressible flow is used.

Doi (1976) subsequently proposed a surface force field featuring a uniform distribution of force on the solid-fluid interface $\Gamma$ :

$$
\boldsymbol{A}_{s}=\left(\frac{\phi}{s} I_{s}-I_{v}\right) \mathbf{1}
$$

The two- and three-point bounds (33) corresponding to this field are:

$$
\begin{aligned}
K \leq K_{s s, 2 \mathrm{pts}} & =\frac{2}{3 \mu} \int_{r=0}^{\infty} r\left(F_{v v}(r)-2 \frac{\phi}{s} F_{v s}(r)+\frac{\phi^{2}}{s^{2}} F_{s s}(r)\right) \mathrm{d} r \\
K \leq K_{s s, 3 \mathrm{pts}} & =\frac{1}{4 \mu} \int_{r=0}^{\infty} \int_{s=0}^{\infty} \int_{u=-1}^{1} \\
& \left(3 u^{2}-1\right) u\left(F_{v v v}(r, s, u)-2 \frac{\phi}{s} F_{v v s}(r, s, u)+\frac{\phi^{2}}{s^{2}} F_{v s s}(r, s, u)\right) \mathrm{d} r \mathrm{~d} s \mathrm{~d} u
\end{aligned}
$$

The two-point bound $K_{s s, 2 \text { pts }}$, known as Doi's bound, has been shown to yield better results than $K_{v v, 2 \text { pts }}$. The three-point bound $K_{s s, 3 p t s}$ has been looked for by previous investigators (see Beasley and Torquato, 1989, p. 206) but is new to the best of our knowledge. As stated by Torquato (1986), Doi had actually suggested a means of obtaining an infinite hierarchy of bounds, involving threepoint information for the next bound in his hierarchy.

Note that the choice of the intensity of the force in the solid-fluid interface for the field (37) is made a priori from the cell equilibrium condition $\boldsymbol{A}_{s} \cdot \boldsymbol{\nabla} \boldsymbol{P} \in$ $\mathcal{F}(\boldsymbol{\nabla} \boldsymbol{P})$ (and similarly for $\boldsymbol{A}_{v}$ ), whereas in previous derivations of Doi's bound this choice is made a posteriori as the only choice ensuring the convergence of the integrals (Rubinstein and Torquato, 1989).

Finally, a new trial field is considered, of surface nature, whose intensity and direction depend on the normal to the solid-fluid interface. For an isotropic medium, the proposed orientation force field corresponds to the following localisation tensor:

$$
\boldsymbol{A}_{o}=\frac{3 \phi}{s} \boldsymbol{I}_{o}-I_{v} \mathbf{1}
$$

where the tensorial surface distribution $\boldsymbol{I}_{o}$ is defined by (3). This choice is motivated by analysis of the solution to particle-in-cells problems (Happel, 1958; Kuwabara, 1959; Boutin, 2000) which have been considered to derive estimates of the permeability of beds of spherical particles. Particle-in-cells problems feature a spherical solid core surrounded by a spherical fluid shell to which appropriate boundary conditions are imposed. One way to retrieve the solution to these problems - although not the one presented by previous investigators is to impose a combination of the two surface force fields (37) and (39) on the boundary of the solid core. In the infinitely dilute case, the contribution of (39) is observed to vanish and (37) alone allows to retrieve the solution to the Stokes 
problem of slow flow past a single sphere in an infinite body. This is the reason why the bounds (38) give a valid asymptotic behaviour in the dilute-limit. The orientation force field is an attempt to deal with non-dilute situations. However, the orientation force field (39) will have to be combined with the surface force field (37) in order to retrieve the correct dilute-limit. The framework of $\S 4.1$ actually ensures that all the previous fields can be combined to derived improve bounds. We hence suggest to consider concentration tensors of force field of the type:

$$
\boldsymbol{A}=\alpha \boldsymbol{A}_{v}+\beta \boldsymbol{A}_{s}+\gamma \boldsymbol{A}_{o} \quad \text { with } \quad \alpha+\beta+\gamma=1
$$

where the last condition is required for the admissibility of the force field: $\boldsymbol{A}$. $\boldsymbol{\nabla} \boldsymbol{P} \in \mathcal{F}(\boldsymbol{\nabla} \boldsymbol{P})$. For isotropic media, the choice of the force field (40) leads to the following expressions of the two- and three-point bounds (33):

$$
K \leq \alpha^{2} K_{v v}+\beta^{2} K_{s s}+\gamma^{2} K_{o o}+2 \alpha \beta K_{s v}+2 \beta \gamma K_{o s}+2 \gamma \alpha K_{v o}
$$

where the following definition are used for the two or three-point bounds:

$$
\begin{aligned}
K_{i j, 2 \mathrm{pts}} & =\frac{1}{6 \mu} \int_{r=0}^{\infty}\left(\mathbf{1}+\boldsymbol{e}_{r} \otimes \boldsymbol{e}_{r}\right):\left\langle\boldsymbol{A}_{i}(\boldsymbol{z}) \cdot \boldsymbol{A}_{j}(\boldsymbol{z}+\boldsymbol{r})\right\rangle r \mathrm{~d} r \\
K_{i j, 3 \mathrm{pts}} & =\frac{1}{4 \mu} \int_{r=0}^{\infty} \int_{s=0}^{\infty} \int_{u=-1}^{1} \boldsymbol{e}_{r} \cdot\left\langle I_{v}(\boldsymbol{z}) \boldsymbol{A}_{i}(\boldsymbol{z}+\boldsymbol{r}) \cdot \boldsymbol{A}_{j}(\boldsymbol{z}+\boldsymbol{s})\right\rangle \cdot \boldsymbol{e}_{s}\left(3 u^{2}-1\right) \mathrm{d} r \mathrm{~d} s \mathrm{~d} u
\end{aligned}
$$

where the subscripts $i$ and $j$ are chosen among $\{v, s, o\}$. The property (32) ensure that the integrands in (42) depend only on $r=|\boldsymbol{r}|$ or $r, s$ and $u$. The terms in the ensemble averaging sign $\langle\bullet\rangle$ in (42) involve simple combinations of the spatial correlation functions introduced in $\S 2$.

The void bounds correspond to $K_{v v}$, i.e. to the case $\beta=\gamma=0$ and the surface ones to $K_{s s, 2 \mathrm{pts}}$, i.e. to the case $\alpha=\gamma=0$. Note that the rectangle terms $K_{v s}, K_{v o}$ and $K_{s o}$ are not bounds themselves. The optimal choice of the weights $\alpha, \beta, \gamma$ under the condition $\alpha+\beta+\gamma=1$ yields the optimal bound:

$$
K \leq K_{\mathrm{opt}}=\frac{K_{v v} K_{s s} K_{o o}+2 K_{v s} K_{s o} K_{o v}-K_{v v} K_{s o}^{2}-K_{s s} K_{o v}^{2}-K_{o o} K_{v s}^{2}}{\Delta}
$$

with $\Delta=2 K_{s o}\left(K_{v s}-K_{v v}\right)+2 K_{v s}\left(K_{o v}-K_{o o}\right)+2 K_{o v}\left(K_{s o}-K_{s s}\right)$

$$
+K_{v v} K_{s s}+K_{s s} K_{o o}+K_{o o} K_{v v}-K_{v s}^{2}-K_{o v}^{2}-K_{s o}^{2}
$$

Interestingly, the evaluation of the combined bounds can be made at no additional computational cost as compared to the surface bound (38) while restricting to $\gamma=0$. The optimal choice is then:

$$
K \leq \frac{K_{v v} K_{s s}-K_{v s}^{2}}{K_{v v}-2 K_{v s}+K_{s s}}
$$

which improves on the void and surface bounds but is weaker than (43). 


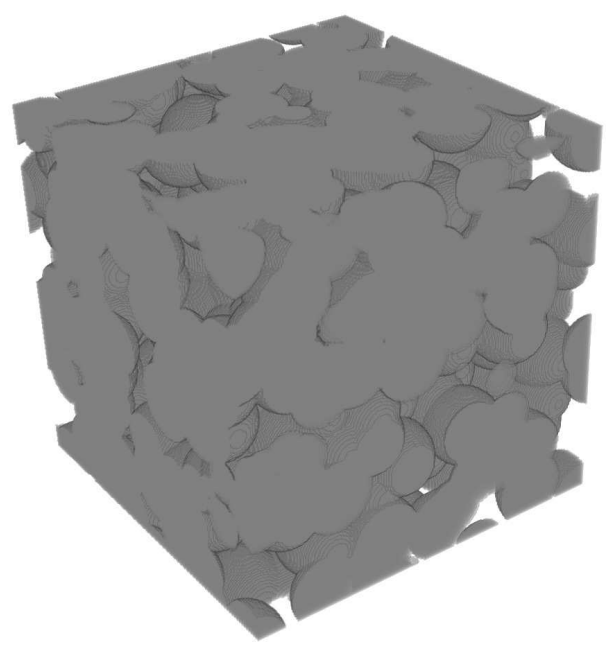

Figure 1: Realisation of a RVE of a Boolean set of 400 spheres with $\phi=21.7 \%$

\section{Permeability of the Boolean model of spheres: results and discussion}

\subsection{Boolean model of spheres}

The bounds derived in $\S 4.4$ are now evaluated for a specific morphological model of random porous media: the Boolean model of spheres (Matheron, 1967). This morphological model is build by randomly positioning fully penetrable spheres according to a uniform spatial distribution, see figure 1 . The present study is restricted to the case where the pore space is the complementary domain of that occupied by the union of the spheres, but the phase interchange case (swiss-cheese like) could be dealt with similarly (Rubinstein and Torquato, 1989; Abdallah et al, 2015). Further, all spheres are supposed to have the same radius $R$. Extensions to non monodisperse sphere size distributions could readily be handled, although to obtain the correct low-density limit, the chosen trial function must depend on the sphere radius (see Given and Stell, 1990; Torquato and $\mathrm{Lu}, 1990$, for the two point surface bound). However, such extensions would be worth of consideration only if the proposed bounds provide accurate results in the easier case presently under consideration.

The Boolean model of spheres is of particular interest since the two- and three-point spatial correlation function involved in the expressions (42) can be derived explicitly, see Online Resource $1^{2}$. Furthermore, this model offers a rather realistic - albeit simplified - representation of actual porous materials such as sandstones, chalks or compacted powders.

\footnotetext{
${ }^{2}$ Appendix A of the present document.
} 


\subsection{Existing estimates and full-field simulations}

In order to assess the quality of the derived bounds, several theoretical bounds and estimates are recalled and full-field simulations are carried out.

\subsubsection{Dilute-limit}

The permeability of the Boolean model of spheres can be evaluated in the dilutelimit $\phi \rightarrow 1$ from Stokes formula (see Childress, 1972; Howells, 1974; Hinch, 1977):

$$
K \underset{\phi \rightarrow 1}{\sim} K_{\text {dilute }}=\frac{2 R^{2}}{9 \mu(1-\phi)}
$$

Since the permeability varies from 0 when $\phi \rightarrow 0$ to $+\infty$ when $\phi \rightarrow 1$, the results on figure 2 appear packed due to the scale dilation. To improve the readability and allow for a more precise discussion, subsequent plots of the obtained permeabilities (figures 3 and 5 ) are scaled by the following factor which is obviously well behaved in the dilute-limit $\phi \rightarrow 1$ :

$$
K_{\text {scale }}=\phi K_{\text {dilute }}
$$

\subsubsection{Effective medium approximations based on Brinkman equa- tion}

To study the flow of a Newtonian fluid in porous media, Brinkman (1949) suggested to combine Darcy's law and Stokes equation as follows:

$$
-\nabla p+\mu \Delta \boldsymbol{v}=\mu \boldsymbol{K}^{-1} \cdot \boldsymbol{v}
$$

The damping force in the r.h.s. corresponds in an approximate way to the average of the forces applied to solid particles to keep them fixed. Based on a modified Stokes formula for the drag on a sphere, Brinkman proposed a selfconsistent approximation of the permeability:

$$
K_{\text {Brinkman }}=\frac{R^{2}}{\mu}\left(\frac{2(2-3 c)}{3\left(3 c+\sqrt{8 c-3 c^{2}}\right)}\right)^{2} \text { with } \quad c=1-\phi
$$

The self-consistent approximation exhibits a percolation threshold $\phi=1 / 3$, below which the permeability vanishes. The Brinkman equations (47) and (48) are actually only valid for low solid concentration $c$; the series expansion of $K_{\text {dilute }} / K_{\text {Brinkman }}$ to $o(c)$ being $1+\frac{3}{\sqrt{2}} \sqrt{c}+\frac{15}{4} c+o(c)$. From a hierarchy of integro-differential equations, Howells (1974); Hinch (1977); Kim and Russel (1985) latter found the series expansion $1+\frac{3}{\sqrt{2}} \sqrt{c}+\frac{135}{64} c \ln (c)+16.456 c+o(c)$ for random arrays of non-penetrable spheres.

To avoid the percolation threshold of the self-consistent scheme and to handle poly-disperse sphere size distributions, Wilkinson (1985) suggested a so-called iterated dilute approximation. The effective medium is build up iteratively as in 


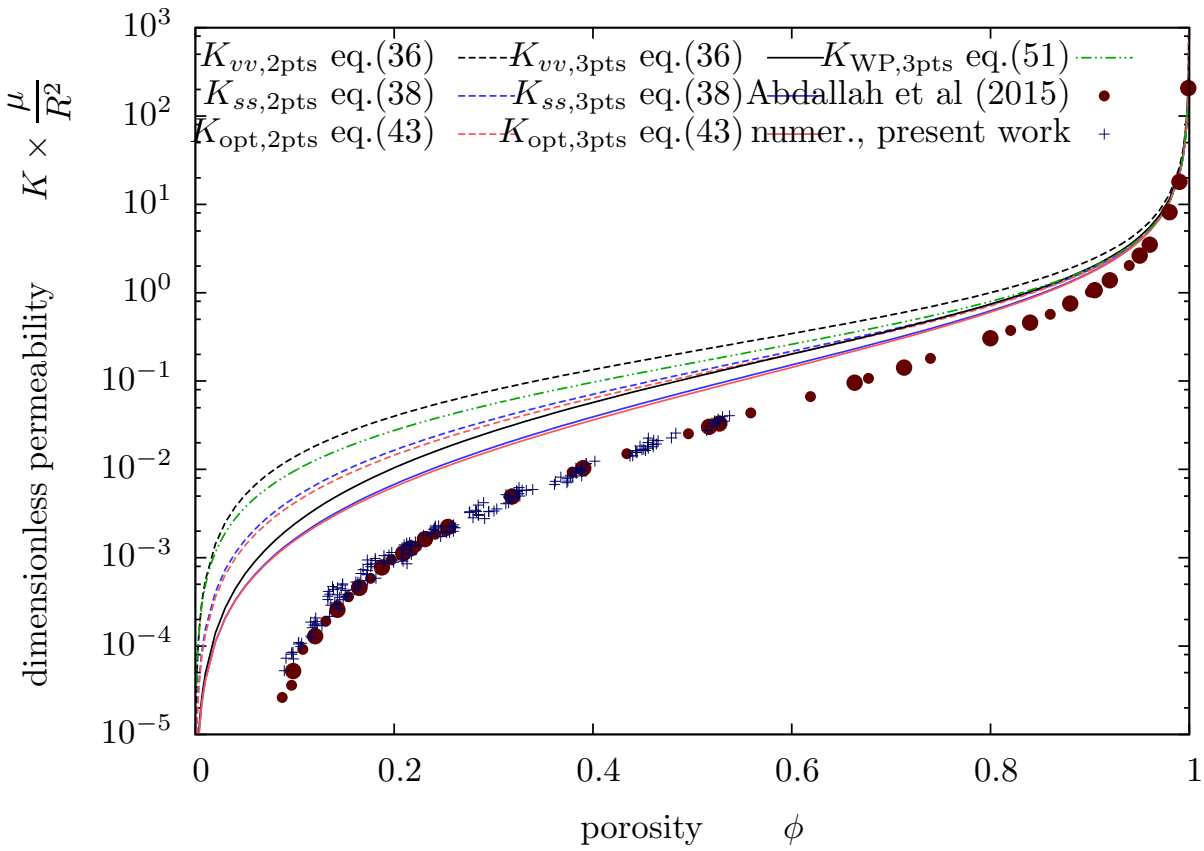

Figure 2: Permeability of Boolean sets of spheres: bounds (lines) eqs. (36), (38), (43) and (51), numerical simulations (points) by Abdallah et al (2015) and additional numerical simulations from the present work (see $\S 5.2 .5$ and figure 1). 
the differential scheme (see e.g. Roscoe, 1952; McLaughlin, 1977), by substituting infinitesimal fractions of solid to the effective Brinkman medium obtained from the previous step. In the case of a mono-disperse sphere size distribution, the procedure leads to the implicit equation:

$$
-\frac{3}{2} \ln \phi=\ln \left(1+\alpha+\alpha^{2} / 3\right)-2 \sqrt{3}\left[\arctan \left(\frac{2 \alpha+3}{\sqrt{3}}\right)-\frac{\pi}{3}\right] \quad \text { with } \quad \alpha=\sqrt{\frac{R^{2}}{K \mu}}
$$

\subsubsection{Particle-in-cell models}

According to the terminology proposed by Dassios et al (1995), particle-in-cell models are based on the solution to a flow problem on a simplified representative pattern of the porous media, of finite extension. The models of Happel (1958) and Kuwabara (1959) belong to this category. They both feature a domain comprising a fixed solid sphere at the centre (the particle), which is surrounded by a spherical shell of Newtonian fluid (the cell). At the boundary of the domain, either uniform velocity boundary conditions are applied (Kuwabara, 1959) or boundary conditions with no tangential stress and a normal stress corresponding to a pressure field with a uniform gradient (Happel, 1958).

Boutin (2000) has shown that the particle-in-cell models may be interpreted as self-consistent models in which the solid particle and the surrounding fluid cell are embedded in an infinite uniform Darcy medium. Two different types of field continuity conditions at the Stokes-Darcy interface are considered : either the velocity is continuous, in which case the model of Kuwabara is retrieved, or the stress vector is continuous while assuming no shear stress in the Darcy domain, in which case the model of Happel is retrieved.

Assuming that the porosity of the particle-in-cell is equal to the one of the modelled porous medium, the following permeability/porosity relationships are obtained from these models (Boutin, 2000) :

$$
\begin{aligned}
K_{\text {Kuwabara }} & =\frac{R^{2}}{\mu} \frac{(1-X)^{3}\left(4+7 X+4 X^{2}\right)}{18 X^{3}\left(1+X+X^{2}+X^{3}+X^{4}\right)} \quad \text { with } \quad X=(1-\phi)^{1 / 3} \\
K_{\text {Happel }} & =\frac{R^{2}}{\mu} \frac{(1-X)^{3}(1+X)\left(2+X+2 X^{2}\right)}{3 X^{3}\left(3+2 X^{5}\right)} \quad \text { }
\end{aligned}
$$

\subsubsection{Bound of Weissberg and Prager (1970)}

In addition to the bounds derived in $\S 4.4$, figures 2,3 and 5 also feature the three point upper bound of Weissberg and Prager (1970):

$$
K \leq K_{W P, 3 \mathrm{pts}}=\frac{2 \phi R^{2}}{-9 \mu \ln \phi}
$$

The bound of Weissberg and Prager (1970) has been established for the Boolean model of spheres by constructing a trial stress field for the variational formulation (22) based on the superposition of stress fields solutions to the Stokes flow 


$\begin{array}{lccccccc}\phi & K_{v v, 2 \mathrm{pts}} & K_{v v, 3 \mathrm{pts}} & K_{s s, 2 \mathrm{pts}} & K_{s s, 3 \mathrm{pts}} & K_{\text {opt }, 2 \mathrm{pts}} & K_{\text {opt,3pts }} & \text { simulations } \\ 0.99 & 26.3254 & 23.4878 & 21.8076 & 21.6422 & 21.7751 & 21.6129 & 18.03 \\ 0.90 & 2.3293 & 1.9146 & 1.8254 & 1.6874 & 1.7976 & 1.6614 & 1.019 \\ 0.80 & 1.0006 & 0.7441 & 0.7341 & 0.6234 & 0.7113 & 0.6030 & 0.306 \\ 0.50 & 0.2170 & 0.1097 & 0.1257 & 0.07887 & 0.1158 & 0.07312 & 0.0255 \\ 0.20 & 0.04009 & 0.01042 & 0.01648 & 0.006764 & 0.01449 & 0.006349 & 0.000956\end{array}$

Table 1: Evaluated bounds and full field simulations by Abdallah et al (2015) of the dimensionless permeability $K \mu / R^{2}$.

past a sphere. By construction, the bound retrieves the correct dilute-limit. As a side remark, let us note that the strategy proposed by Beasley and Torquato (1989) to derive new bounds on the permeability of random arrays of spheres is more closely linked to the approach of Weissberg and Prager (1970) than originally thought by the authors. Although it had been applied to a random model of impenetrable spheres in the original paper, a simple adaptation of this strategy to penetrable sphere can be shown to also lead to (51).

\subsubsection{Full-field simulations}

The bounds are compared in figure 2 to full field simulations performed on several realisations of representative volume elements of Boolean sets of spheres over the whole range of porosity. In addition to the numerical results of Abdallah et al (2015), a series of dedicated simulations have been performed using the numerical scheme presented in Bignonnet and Dormieux (2014). The latter simulations have been performed on a $256^{3}$-voxel discretization grid with sphere radii of 24 voxels. The simulated microstructures feature up to several hundreds of spheres, depending on the target porosity. Figure 1 presents an example of simulated microstructure. Both numerical methods lead to similar results and will be considered as a reference to assess the accuracy of the bounds.

\subsection{Results}

Figures 3 illustrates that for the same force field, three point bounds provide a sizeable improvement on their two point counterparts. Among three point bounds, the Weissberg and Prager (1970) bound is the weakest for most porosities, apart from the void bound $K_{v v, 3 p t s}$ in the dilute-limit $\phi \rightarrow 1$. The bounds (36) based on the void force field over-estimate the permeability are indeed ill-behaved in the dilute-limit, by a factor of $6 / 5$ for the two-point bound and $27 / 25$ for the three-point bound, whereas the other bounds have a valid asymptotic behaviour. The void bounds $K_{v v}$ are systematically weaker than the surface bounds $K_{s s}$, in their two- or three-point versions. As expected, the optimal bounds $K_{\mathrm{opt}}$ are sharper than the surface bounds. However, the improvement is minor. This is rather disappointing since the orientation force field is well activated as indicated by sizeable positive values of the optimal choice of 


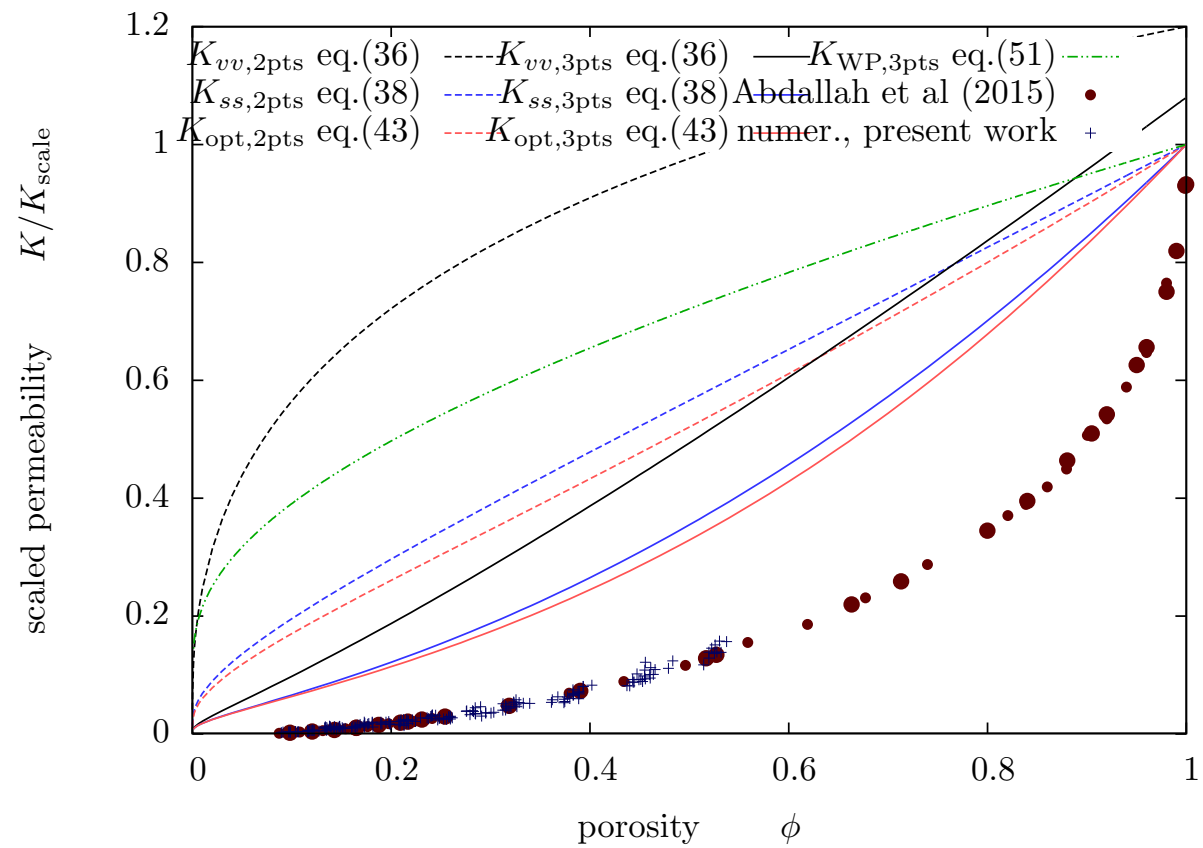

Figure 3: Permeability of Boolean sets of spheres, scaled by (46): bounds (lines) eqs. (36), (38), (43) and (51), numerical simulations (points) by Abdallah et al (2015) and additional numerical simulations from the present work (see $§ 5.2 .5$ and figure 1). 
optimal weights for $\boldsymbol{A}_{v}, \boldsymbol{A}_{s}$ and $\boldsymbol{A}_{o}$

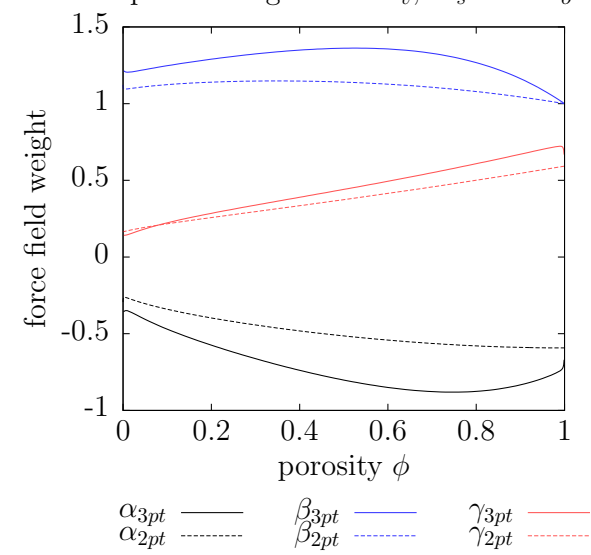

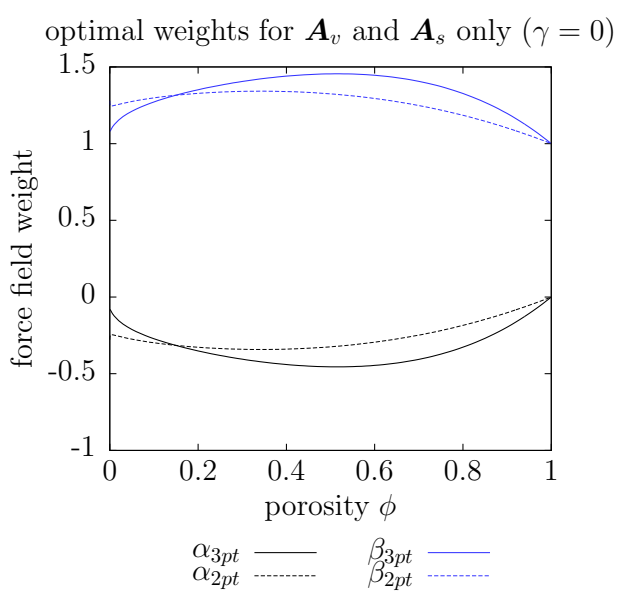

Figure 4: Optimal values of the force field weights $\alpha, \beta, \gamma$ in (40)

the weight $\gamma$ in figure 4a. The bounds (44) are not displayed as they lie within $K_{s s}$ and $K_{\mathrm{opt}}$, which are already close. The optimal choice of the weights $\alpha$ and $\beta$ under the constraint $\gamma=0$ for the combined force field (40) clearly indicates that the surface force field takes over the void force field (see figure $4 \mathrm{~b}$ ).

Next, the bounds are quantitatively compared to the full field simulations. Table 1 indicates that the weakest bounds such as $K_{v v, 2 p t s}$ may not be used as reliable estimates of the permeability since they differ from simulation data by more than one order of magnitude. The best bounds, namely $K_{s s, 3 p t s}$ and $K_{\text {opt,3pts }}$ are better behaved but overestimate the simulation data in the nondilute regime, by a factor 2 at $80 \%$ of porosity, 3 at $\phi=50 \%$ and 7 at $\phi=20 \%$. As will be discussed later, it is already satisfactory that these bounds deliver the proper order of magnitude since even estimates fail to provide accurate results in the non-dilute regime. For example, the bound $K_{s s, 3 p t s}$ improves on the iterated dilute approximation of Wilkinson (1985) for porosities below $30 \%$.

\subsection{Discussion}

Among three point bounds, the force field based bounds (33) improve on the bound based on the solution to the Stokes flow past a sphere (51). By construction, the Weissberg and Prager (1970) bound indeed only conveys statistical information about the position of the sphere centres. Since the latter are uncorrelated in the Boolean model, such information is rather poor. On the contrary, the volume and surface force field based bounds involve a richer information, namely three-point correlation functions involving the fluid phase and the solidfluid interface.

An alternative point of view on the force field based bounds is that they enforce particular constraints on the velocity field. Referring to the auxiliary problem (23), the uniform volume force field corresponds to the Lagrange mul- 


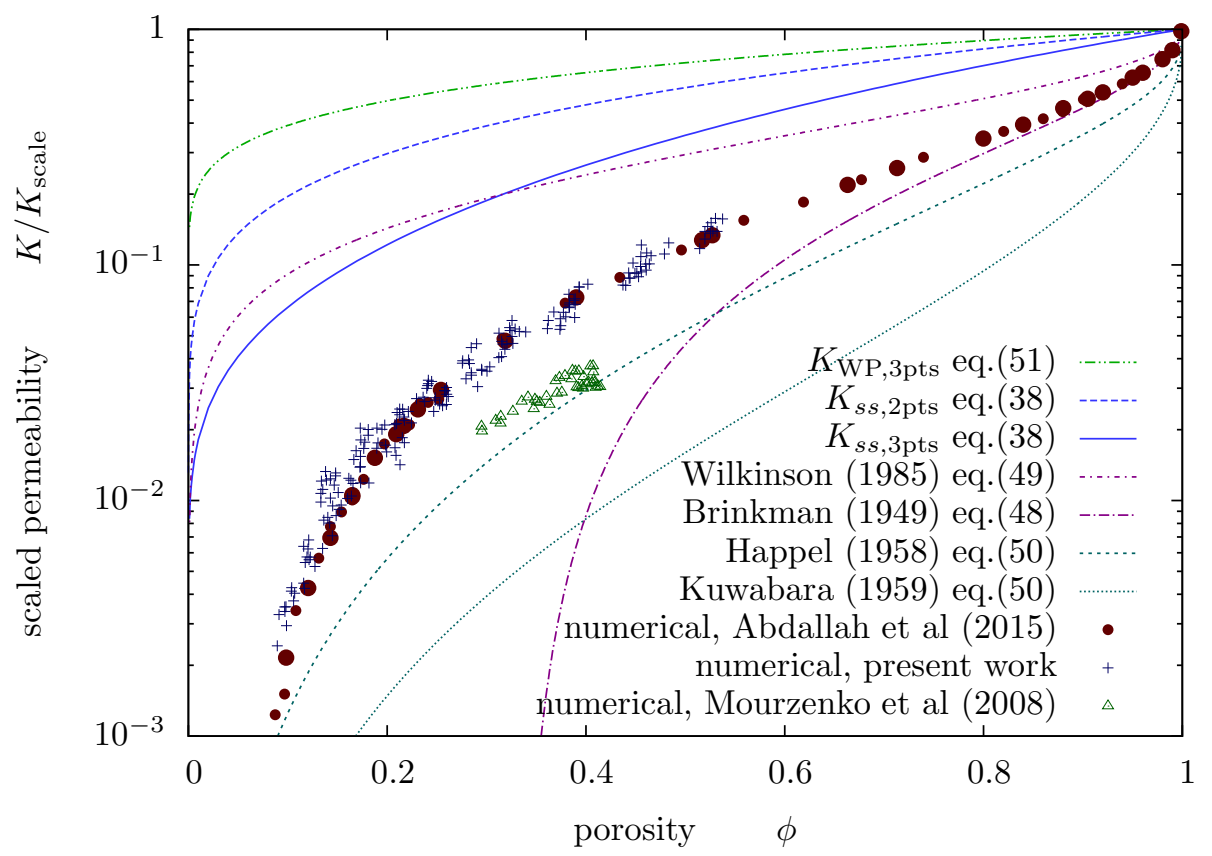

Figure 5: Permeability of Boolean sets of spheres, scaled by (46): bounds and estimates (lines), numerical simulations (points) by Abdallah et al (2015) and additional numerical simulations from the present work (see $\S 5.2 .5$ and figure 1). In turn, the simulations of Mourzenko et al (2008) correspond to loose packings of polydisperse unpenetrable spheres.

tiplier of the constraint : "the average of the velocity over the domain occupied by the solid phase is null" for a flow in a uniform fluid. The uniform surface force field corresponds to the Lagrange multiplier of the constraint : "the average of the velocity over the domain occupied the solid-fluid interface is null". When both of these constraints are imposed, figure $4 \mathrm{~b}$ and the proximity of the bounds (38) and (44) indicate that the latter constraint is the most efficient. The reason is that the interface constraint is related - although in a diminished version - to the no-slip constraint at all points of the solid-fluid interface in the actual problem.

Although the $K_{W P}, K_{s s}$ and $K_{\text {opt }}$ bounds retrieve the correct asymptotic behaviour in the dilute-limit, figures 3 and 5 illustrate that none of them reproduce the non-analytic higher order terms in the series expansion of $K / K_{\text {dilute }}$. Contrarily, denoting by $c=1-\phi$ the solid phase concentration, effective medium approximations based on Brinkman equation evidence a higher order term in $\sqrt{c}$ for $c \rightarrow 0$. The underlying idea of the Brinkman-based approaches is that, from the point of view of a solid sphere, the near field involves mainly viscous forces and follows Stokes equation, while due to the shielding effect of the other 
spheres, the far field is pressure gradient driven and follows Darcy equation.

In the light of the work of Boutin (2000), particle-in-cell models also correspond to a similar idea of decomposition of the far and near fields (see §5.2.3). At low porosities, the Happel particle-in-cell model provides the best permeability estimate among all presented models but underestimates the permeability. At high porosities, figure 5 illustrates that the particle-in-cell models lead to a too stiff decrease of the permeability with increasing solid concentration, with a second order term in $\sqrt[3]{c}$ instead of $\sqrt{c}$. As discussed by Saffman (1973), the $\sqrt[3]{c}$ term which also arises for periodic cubic arrays is due to the existence of a lengthscale associated with the cell. The numerical results of Mourzenko et al (2008) indicate that for intermediate porosities, the Happel estimate is actually closer to the permeability of loose packings of non-overlapping spheres, for which the porosity is more evenly distributed around the solid particles than for the Boolean model.

As suggested by Saffman (1973), the $\sqrt{c}$ term for random fixed arrays of fixed spheres arises because the forces which keep the spheres fix are random. However, the present choices of force field are deterministic, in that the values of the tested force fields are uniform within a phase (solid, fluid or interface) and do not depend on the position of other particles in the neighbourhood. To us, this is precisely where an improvement of the bounds is to be sought. Unfortunately from a practical point of view, spatial correlation of higher order will likely arise while following that way.

\section{Conclusion}

New bounds on the permeability of random porous media have been proposed, improving earlier versions and clarifying their links. All these bounds are expressed as a function of statistical descriptors of the morphology of the pore space, namely two- and three-point correlation functions. The spatial correlation functions involved in the bounds are explicitly derived for the Boolean model of spheres. From a practical point of view, the implementation of the proposed three-point bounds is somewhat involved since it requires, in addition to the knowledge of three-point correlation functions involving the fluid phase and the solid-fluid interface, a threefold numerical integration.

To the best of our knowledge, the newly derived three-point bound based on the surface force field, which is seen as a higher order version of the Doi bound, is the first bound that predicts the correct order of magnitude as compared to numerical simulation data. The optimal bounds including the surface orientation force field are very close to the simpler bounds based on the sole surface force field. Since the derivation of such bounds which feature information about the local orientation of the solid-fluid interface is much more involved, they do not

offer an interesting accuracy/complexity ratio. Note that applications of the bounds derived herein to other morphological models than the Boolean model of spheres are still prospective and would likely be computationally involved.

Nonetheless, there is still room for major improvements of the surface field 
based bounds. The proposed strategy relies on piece-wise uniform force fields combined with the Green operator for an infinite body governed by Stokes equation, and thus loses the random character of the force on solid particles. In particular, the non-analytic behaviour of the series expansion of the permeability at low solid concentration is not retrieved.

\section{References}

Abdallah B, Willot F, Jeulin D (2015) Stokes flow through a boolean model of spheres: Representative volume element. Transport in Porous Media 109(3):711-726

Auriault JL (2002) Upscaling heterogeneous media by asymptotic expansions. J Eng Mech 128(8):817-822

Auriault JL, Sanchez-Palencia E (1977) Etude du comportement macroscopique d'un milieu poreux saturé déformable. J Mécanique 16:575-603

Auriault JL, Geindreau C, Boutin C (2005) Filtration law in porous media with poor separation of scales. Transport in Porous Media 60(1):89-108

Beasley JD, Torquato S (1989) New bounds on the permeability of a random array of spheres. Physics of Fluids 1(2):199-207

Beran M (1965) Use of a variational approach to determine bounds for the effective permittivity of a random medium. Nuovo Cimento 38:771-782

Berryman JG (1983) Computing variational bounds for flow through random aggregates of sphere. Journal of Computational Physics 52(1):142-162

Berryman JG, Milton GW (1985) Normalization constraint for variational bounds on fluid permeability. J Chem Phys 83, 754 83:754-760

Bignonnet F, Dormieux L (2014) FFT-based bounds on the permeability of complex microstructures. International Journal for Numerical and Analytical Methods in Geomechanics 38(16):1707-1723

Boutin C (2000) Study of permeability by periodic and self-consistent homogenisation. European Journal of Mechanics A/Solids 19:603-632

Brinkman HC (1949) A calculation of the viscous force exerted by a flowing fluid on a dense swarm of particles. Appl Sci Res 1(1):27

Childress S (1972) Viscous flow past a random array of spheres. J Chem Phys $56(6): 2527-2539$

Chiu SN, Stoyan D, Kendall WS, Mecke J (2013) Stochastic geometry and its applications. John Wiley \& Sons 
Dassios G, Hadjinicolaou M, Coutelieris F, Payatakes A (1995) Stokes flow in spheroidal particle-in-cell models with happel and kuwabara boundary conditions. Int J Engng Sci 33:1465-1490

Doi M (1976) A new variational approach to the diffusion and flow problem in porous media. J Phys Soc Japan 40:567-572

Ene H, Sanchez-Palencia E (1975) Equations et phénomènes de surface pour l'écoulement dans un modèle de milieu poreux. Journal de Mécanique pp $73-108$

Gibson KD, Scheraga HA (1987) Volume of the intersection of three spheres of unequal size. A simplified formula. J Phys Chem 91:4121-4122

Given JA, Stell G (1990) Improved permeability bounds for highly polydisperse materials. Physics of Fluids A: Fluid Dynamics 2:1929-1936

Happel J (1958) Viscous flow in multiparticle systems: slow motion of fluids relative to beds of spherical particles. AIChE J 4:197-201

Hashin Z, Shtrikman S (1962) On some variational principles in anisotropic and nonhomogeneous elasticity. Journal of the Mechanics and Physics of Solids 10(4):335-342

Hinch EJ (1977) An averaged-equation approach to particle interactions in a fluid suspension. J Fluid Mech 83(4):695-720

Howells I (1974) Drag due to the motion of a newtonian fluid through a sparse random array of small fixed rigid objects. J Fluid Mech 64(3):449-476

Kim S, Russel WB (1985) Modelling of porous media by renormalization of the Stokes equations. Journal of Fluid Mechanics 154:269-286, DOI 10.1017/ S0022112085001525

Kuwabara S (1959) The forces experienced by randomly distributed parallel circular cylinders or spheres in a viscous flow at small reynolds numbers. J Phys Soc Jpn 14:527-532

Matheron G (1967) Élements pour une théorie des milieux poreux. Paris, Masson

Matheron G (1975) Random sets and integral geometry, volume 1. Wiley New York

McLaughlin R (1977) A study of the differential scheme for composite materials. International Journal of Engineering Science 15(4):237-244

Mourzenko VV, Thovert JF, Vizika, Adler PM (2008) Geometrical and transport properties of random packings of polydisperse spheres. Phys Rev E $77(6): 066,306$ 
Piessens R, Doncker-Kapenga ED, Überhuber CW (1983) QUADPACK: a subroutine package for automatic integration. Springer

Ponte Castañeda P, Willis JR (1995) The effect of spatial distribution on the effective behavior of composite materials and cracked media. Journal of the Mechanics and Physics of Solids 43(12):1919 - 1951

Prager S (1961) Viscous flow through porous media. Phys Fluids 4:1477-1482

Röding M, Svensson P, Lorén N (2017) Functional regression-based fluid permeability prediction in monodisperse sphere packings from isotropic two-point correlation functions. Comp Mater Sci 134:126-131

Roscoe R (1952) The viscosity of suspensions of rigid spheres. Brit J Appl Phys 3(8):267-269

Rubinstein J, Torquato S (1989) Flow in random porous media: mathematical formulation, variational principles, and rigorous bounds. J Fluid Mech 206:2546

Sab K (1994) Homogenization of non-linear random media by a duality method. Application to plasticity. Asymptotic Analysis 9(4):311-336

Saffman PG (1973) On the settling speed of free and fixed suspensions. Stud Appl Math 52(2):115-127

Torquato S (1986) Microstructure characterization and bulk properties of disordered two-phase media. Journal of Statistical Physics 45(5/6):843-873

Torquato S (2002) Random heterogeneous materials: microstructure and macroscopic properties. Springer

Torquato S, Beasley JD (1987) Bounds on the permeability of a random array of partially penetrable spheres. Physics of Fluids (1958-1988) 30:633-641

Torquato S, Lu B (1990) Rigorous bounds on the fluid permeability: Effect of polydispersivity in grain size. Physics of Fluids A: Fluid Dynamics 2:487-490

Weissberg HL, Prager S (1970) Viscous flow through porous media. III. Upper bounds on the permeability for a simple random geometry. Physics of Fluids 13(12):2958-2965

Whitaker S (1986) Flow in porous media I: A theoretical derivation of darcy's law. Transport Porous Med 1(1):3-25

Wiegmann A (2007) Computation of the permeability of porous materials from their microstructure by FFF-Stokes. Berichte des Fraunhofer ITWM 129

Wilkinson D (1985) Modified drag theory of permeability. The Physics of Fluids 28(4):1015-1022 
Willis JR (1977) Bounds and self-consistent estimates for the overall properties of anisotropic composites. J Mech Phys Solids 25:185-202

Willot F, Abdallah B, Jeulin D (2016) The permeability of boolean sets of cylinders. Oil \& Gas Science and Technology - Revue d'IFP Energies nouvelles $71(4)$ 
The following purely technical aspects which are required to evaluate the bounds on the permeability presented in the above article were provided as "Electronic Supplementary Material" in the published version.

\section{A Spatial correlation functions of the Boolean model of spheres}

A systematic derivation of all the required spatial correlation functions for the Boolean model of spheres is presented in detail for the sake of completeness. Some results for the scalar correlation functions have already been provided by previous authors (see e.g. Torquato, 1986, 2002), but the tensorial correlation functions which involve additional information about the orientation of the normal to the solid-fluid interface are new.

\section{A.1 Notations}

A Representative Volume Element (RVE) $\Omega$ of a porous medium comprises a fluid phase (or void phase) in the domain $\Omega_{f}$ and a solid phase in the domain $\Omega_{s}$, such that $\Omega_{f} \cup \Omega_{s}=\Omega$. The solid-fluid interface is denoted $\Gamma$, and its local unit normal $\boldsymbol{n}$ (see figure 6). Let us introduce the following indicator functions $I_{v}, I_{s}$ and $\boldsymbol{I}_{o}$ such that for any field $f$ :

$$
\begin{aligned}
\int_{\Omega} f(\boldsymbol{z}) I_{v}(\boldsymbol{z}) \mathrm{d} V_{z} & =\int_{\Omega_{f}} f(\boldsymbol{z}) \mathrm{d} V_{z} \\
\int_{\Omega} f(\boldsymbol{z}) I_{s}(\boldsymbol{z}) \mathrm{d} V_{z} & =\int_{\Gamma} f(\boldsymbol{z}) \mathrm{d} S_{z} \\
\int_{\Omega} f(\boldsymbol{z}) \boldsymbol{I}_{o}(\boldsymbol{z}) \mathrm{d} V_{z} & =\int_{\Gamma} f(\boldsymbol{z}) \boldsymbol{n}(\boldsymbol{z}) \otimes \boldsymbol{n}(\boldsymbol{z}) \mathrm{d} S_{z}
\end{aligned}
$$

where $\boldsymbol{z}$ denotes the position vector in the RVE. In the following developments, the random media is assumed stationary and ergodic.

Based on these three indicator functions, several types of one-point $(\boldsymbol{z})$, twopoint $(\boldsymbol{z}, \boldsymbol{z}+\boldsymbol{r})$ and three-point $(\boldsymbol{z}, \boldsymbol{z}+\boldsymbol{r}, \boldsymbol{z}+\boldsymbol{s})$ correlation functions are defined as follows:

$$
\begin{aligned}
F_{i} & =\left\langle I_{i}(\boldsymbol{z})\right\rangle \\
F_{i j}(\boldsymbol{r}) & =\left\langle I_{i}(\boldsymbol{z}) I_{j}(\boldsymbol{z}+\boldsymbol{r})\right\rangle \\
F_{v i j}(\boldsymbol{r}, \boldsymbol{s}) & =\left\langle I_{v}(\boldsymbol{z}) I_{i}(\boldsymbol{z}+\boldsymbol{r}) I_{j}(\boldsymbol{z}+\boldsymbol{s})\right\rangle
\end{aligned}
$$

where the subscripts $i$ and $j$ are chosen among $\{v, s, o\}$ and the dot product is to be used between $\boldsymbol{I}_{o}$ and $\boldsymbol{I}_{o}$ whenever $i=j=o$.

Note that for two surfaces $S_{1}$ and $S_{2}$ of $\Omega$, whose unit normals are respectively denoted $\boldsymbol{n}_{1}$ and $\boldsymbol{n}_{2}$ and whose indicator (surface) distributions are denoted $I_{1}$ and $I_{2}$, the product $I_{1} I_{2}$ is a line distribution such that:

$$
\forall f, \quad \int_{\Omega} I_{1}(\boldsymbol{z}) I_{2}(\boldsymbol{z}) f(\boldsymbol{z}) \mathrm{d} V_{z}=\int_{S_{1} \cap S_{2}} f(\boldsymbol{z}) \frac{\mathrm{d} L_{z}}{\sqrt{1-\left(\boldsymbol{n}_{1} \cdot \boldsymbol{n}_{2}\right)^{2}}}
$$




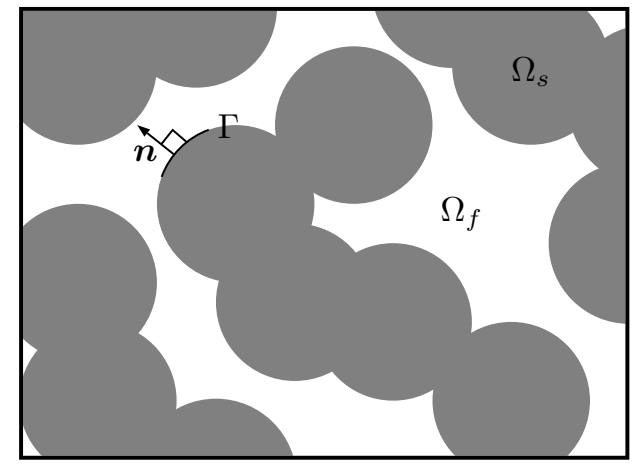

Figure 6: Schematic Representative Volume Element $\Omega$ of a porous medium

where the right hand side is a line integral over the intersection of $S_{1}$ and $S_{2}$, which may diverge if the two surfaces are tangent.

\section{A.2 Boolean model of spheres}

The Boolean model of spheres is built on a Poisson point process of rate $\rho$. For more details, the reader is referred to reference books on random set theory (see detailed presentations in Matheron, 1975; Torquato, 2002; Chiu et al, 2013). Considering a RVE, a collection of $N$ random points (or germs) $\left(\boldsymbol{c}_{i}\right)_{i=1 \ldots N}$ are first randomly positioned in $\Omega$. The rate $\rho$ corresponds number of these points per unit volume:

$$
\rho=\frac{N}{|\Omega|}
$$

In the following developments, the limiting case $N \rightarrow \infty$ and $\Omega \rightarrow \mathbb{R}^{3}$ for prescribed values of $\rho$ is considered. The solid phase is then defined as the union of the spheres centred at the points $\boldsymbol{c}_{i}$, and the fluid phase as the complementary domain. The solid phase is thus made of potentially overlapping spheres of radius $R$. The roles of the solid and fluid phases could be interchanged, resulting in a different morphology of the pore space. This phase interchange alternative will not be dealt here, but has been studied by Rubinstein and Torquato (1989); Abdallah et al (2015) among others.

Since the positions of the sphere centres are uncorrelated in the Boolean model, the required n-point correlation functions will now be derived using the following ensemble averaging rule, which amounts to average over all positions of the sphere centres $\left(\boldsymbol{c}_{i}\right)_{i=1 \ldots N}$ :

$$
\langle\bullet\rangle=\frac{1}{|\Omega|^{N}} \int_{\Omega} \ldots \int_{\Omega} \bullet \mathrm{d} V_{c_{1}} \ldots \mathrm{d} V_{c_{n}}
$$




\section{A.3 Decomposition of the void, interface and oriented in- terface indicator functions}

The void, interface and oriented interface indicator functions for a single sphere of radius $R$ centred at the origin, respectively denoted by $V, S$ and $\boldsymbol{O}$, are defined such as for any field $f$ :

$$
\begin{aligned}
\int_{\Omega} f(\boldsymbol{z}) V(\boldsymbol{z}) \mathrm{d} V_{z} & =\int_{\|\boldsymbol{z}\| \geq R} f(\boldsymbol{z}) \mathrm{d} V_{z} \\
\int_{\Omega} f(\boldsymbol{z}) S(\boldsymbol{z}) \mathrm{d} V_{z} & =\int_{\|\boldsymbol{z}\|=R} f(\boldsymbol{z}) \mathrm{d} S_{z} \\
\int_{\Omega} f(\boldsymbol{z}) \boldsymbol{O}(\boldsymbol{z}) \mathrm{d} V_{z} & =\int_{\|\boldsymbol{z}\|=R} f(\boldsymbol{z}) \boldsymbol{n}(\boldsymbol{z}) \otimes \boldsymbol{n}(\boldsymbol{z}) \mathrm{d} S_{z}
\end{aligned}
$$

Using these notations, the indicator functions of the void phase $I_{v}$, of the solidfluid interface $I_{s}$ and its oriented version $\boldsymbol{I}_{o}$ defined by (52) can be generated as functions of the positions $\left(\boldsymbol{c}_{i}\right)_{i=1 \ldots N}$ of the sphere centres:

$$
\begin{aligned}
I_{v}(\boldsymbol{z}) & =\prod_{i=1}^{N} V\left(\boldsymbol{z}-\boldsymbol{c}_{i}\right) \\
I_{s}(\boldsymbol{z}) & =\sum_{j=1}^{N}\left[S\left(\boldsymbol{z}-\boldsymbol{c}_{j}\right) \prod_{i \neq j} V\left(\boldsymbol{z}-\boldsymbol{c}_{i}\right)\right] \\
\boldsymbol{I}_{o}(\boldsymbol{z}) & =\sum_{j=1}^{N}\left[\boldsymbol{O}\left(\boldsymbol{z}-\boldsymbol{c}_{j}\right) \prod_{i \neq j} V\left(\boldsymbol{z}-\boldsymbol{c}_{i}\right)\right]
\end{aligned}
$$

\section{A.4 One-point correlation functions}

The one-point correlation function of the void phase $F_{v}$, equal to the porosity $\phi=\left|\Omega_{f}\right| /|\Omega|$, may be derived as a function of the rate $\rho=N /|\Omega|$ of the Poisson point process as follows:

$$
\begin{aligned}
F_{v} & =\frac{1}{|\Omega|^{N}} \int_{\Omega} \ldots \int_{\Omega} \prod_{i=1}^{N} V\left(\boldsymbol{z}-\boldsymbol{c}_{i}\right) \mathrm{d} V_{c_{1}} \ldots \mathrm{d} V_{c_{n}} \\
& =\prod_{i=1}^{N} \frac{1}{|\Omega|} \int_{\Omega} V\left(\boldsymbol{x}_{i}\right) \mathrm{d} V_{x_{i}} \text { with } \boldsymbol{x}_{i}=\boldsymbol{z}-\boldsymbol{c}_{i} \\
& =\left(1-\frac{\rho M_{v}}{N}\right)^{N} \underset{N \rightarrow \infty}{\longrightarrow} \exp \left(-\rho M_{v}\right)
\end{aligned}
$$

where the independence of the positions of the centres of the spheres has been used and $M_{v}$ is the volume of a sphere of radius $R$ :

$$
M_{v}=\int 1-V(\boldsymbol{x}) \mathrm{d} V_{x}=\frac{4 \pi R^{3}}{3}
$$


The one-point correlation function of the interface $F_{s}$ is equal to the specific surface area $s=|\Gamma| /|\Omega|$. Proceeding as for (59):

$$
\begin{aligned}
F_{s} & =\frac{1}{|\Omega|^{N}} \int_{\Omega} \ldots \int_{\Omega} \sum_{j=1}^{N}\left[S\left(\boldsymbol{z}-\boldsymbol{c}_{j}\right) \prod_{i \neq j} V\left(\boldsymbol{z}-\boldsymbol{c}_{i}\right)\right] \mathrm{d} V_{c_{1}} \ldots \mathrm{d} V_{c_{n}} \\
& =\sum_{j=1}^{N}\left[\frac{1}{|\Omega|} \int_{\Omega} S\left(\boldsymbol{x}_{j}\right) \mathrm{d} V_{x_{j}} \prod_{i \neq j} \frac{1}{|\Omega|} \int_{\Omega} V\left(\boldsymbol{x}_{i}\right) \mathrm{d} V_{x_{i}}\right] \\
& =N \frac{\rho}{N} M_{s}\left(1-\frac{\rho M_{v}}{N}\right)^{N-1} \underset{N \rightarrow \infty}{\longrightarrow} \rho M_{s} \exp \left(-\rho M_{v}\right)
\end{aligned}
$$

where $M_{s}$ is the surface area of a sphere of radius $R$ :

$$
M_{s}=\int S(\boldsymbol{x}) \mathrm{d} V_{x}=4 \pi R^{2}
$$

\section{A.5 Two-point correlation functions}

Since the centres of the spheres follow a random Poisson process, the two-point correlation functions indicate a decorrelation as soon as the distance $r$ between the two points is greater than the diameter of the spheres. It is then convenient to express the results as a function of the dimensionless parameter $\eta$ :

$$
\eta=\frac{r}{2 R}
$$

\section{A.5.1 Scalar two-point correlation functions}

Let us introduce the following geometrical measures on the union of two spheres:

$$
\begin{array}{r}
M_{v v}(r)=\int 1-V(\boldsymbol{x}) V(\boldsymbol{x}+\boldsymbol{r}) \mathrm{d} V_{x}=M_{v} \times \begin{cases}1+\frac{3}{2} \eta-\frac{1}{2} \eta^{3} & \text { if } \eta \leq 1 \\
2 & \text { if } \eta>1\end{cases} \\
M_{s v}(r)=\int S(\boldsymbol{x}) V(\boldsymbol{x}+\boldsymbol{r}) \mathrm{d} V_{x}=M_{s} \times \begin{cases}\frac{1+\eta}{2} & \text { if } \eta \leq 1 \\
1 & \text { if } \eta>1\end{cases} \\
M_{s s}(r)=\int S(\boldsymbol{x}) S(\boldsymbol{x}+\boldsymbol{r}) \mathrm{d} V_{x}=M_{s} \times \begin{cases}\frac{1}{4 R \eta} & \text { if } \eta \leq 1 \\
0 & \text { if } \eta>1\end{cases}
\end{array}
$$

$M_{v v}(r)$ is the volume of the union of two spheres whose centres are distant of $r . M_{s v}(r)$ is the surface area of the sphere of radius $R$ centred at the origin, at the exclusion of the sphere of radius $R$ centred at $\boldsymbol{r} . M_{s s}(r)$ scales as a length 
and is determined using the property (7). Using these notations, the two-point correlation functions are given by:

$$
\begin{aligned}
& F_{v v}(\boldsymbol{r})=\frac{1}{|\Omega|^{N}} \int_{\Omega} \ldots \int_{\Omega} \prod_{i=1}^{N} V\left(\boldsymbol{x}_{i}\right) V\left(\boldsymbol{x}_{i}+\boldsymbol{r}\right) \mathrm{d} V_{x_{1}} \ldots \mathrm{d} V_{x_{n}} \\
& =\prod_{i=1}^{N} \frac{1}{|\Omega|} \int_{\Omega} V\left(\boldsymbol{x}_{i}\right) V\left(\boldsymbol{x}_{i}+\boldsymbol{r}\right) \mathrm{d} V_{x_{i}} \\
& =\left(1-\frac{\rho M_{v v}(r)}{N}\right)^{N} \underset{N \rightarrow \infty}{\longrightarrow} \exp \left(-\rho M_{v v}(r)\right) \\
& F_{s v}(\boldsymbol{r})=\frac{1}{|\Omega|^{N}} \int_{\Omega} \ldots \int_{\Omega}\left[\sum_{j=1}^{N} S\left(\boldsymbol{x}_{j}\right) \prod_{i \neq j} V\left(\boldsymbol{x}_{i}\right)\right] \prod_{k=1}^{N} V\left(\boldsymbol{x}_{k}+\boldsymbol{r}\right) \mathrm{d} V_{x_{1}} \ldots \mathrm{d} V_{x_{n}} \\
& =\sum_{j=1}^{N}\left[\frac{1}{|\Omega|} \int_{\Omega} S\left(\boldsymbol{x}_{j}\right) V\left(\boldsymbol{x}_{j}+\boldsymbol{r}\right) \mathrm{d} V_{x_{j}} \prod_{i \neq j} \frac{1}{|\Omega|} \int_{\Omega} V\left(\boldsymbol{x}_{i}\right) V\left(\boldsymbol{x}_{i}+\boldsymbol{r}\right) \mathrm{d} V_{x_{i}}\right] \\
& =N \frac{\rho}{N} M_{s v}(r)\left(1-\frac{\rho M_{v v}(r)}{N}\right)^{N-1} \underset{N \rightarrow \infty}{\longrightarrow} \rho M_{s v}(r) \exp \left(-\rho M_{v v}(r)\right) \\
& F_{s s}(\boldsymbol{r})=\frac{1}{|\Omega|^{N}} \int_{\Omega} \ldots \int_{\Omega}\left[\sum_{j=1}^{N} S\left(\boldsymbol{x}_{j}\right) \prod_{i \neq j} V\left(\boldsymbol{x}_{i}\right)\right]\left[\sum_{k=1}^{N} S\left(\boldsymbol{x}_{k}+\boldsymbol{r}\right) \prod_{\ell \neq k} V\left(\boldsymbol{x}_{\ell}+\boldsymbol{r}\right)\right] \mathrm{d} V_{x_{1}} \ldots \mathrm{d} V_{x_{n}} \\
& =\sum_{j=1}^{N} \frac{1}{|\Omega|} \int_{\Omega} S\left(\boldsymbol{x}_{j}\right) S\left(\boldsymbol{x}_{j}+\boldsymbol{r}\right) \mathrm{d} V_{x_{j}} \prod_{i \neq j} \frac{1}{|\Omega|} \int_{\Omega} V\left(\boldsymbol{x}_{i}\right) V\left(\boldsymbol{x}_{i}+\boldsymbol{r}\right) \mathrm{d} V_{x_{i}} \\
& +\sum_{j=1}^{N} \sum_{k \neq j}\left[\frac{1}{|\Omega|} \int_{\Omega} S\left(\boldsymbol{x}_{j}\right) V\left(\boldsymbol{x}_{j}+\boldsymbol{r}\right) \mathrm{d} V_{x_{j}} \frac{1}{|\Omega|} \int_{\Omega} S\left(\boldsymbol{x}_{k}+\boldsymbol{r}\right) V\left(\boldsymbol{x}_{k}\right) \mathrm{d} V_{x_{k}}\right. \\
& \left.\prod_{i \neq j, k} \frac{1}{|\Omega|} \int_{\Omega} V\left(\boldsymbol{x}_{i}\right) V\left(\boldsymbol{x}_{i}+\boldsymbol{r}\right) \mathrm{d} V_{x_{i}}\right] \\
& =N \frac{\rho}{N} M_{s s}(r)\left(1-\frac{\rho M_{v v}(r)}{N}\right)^{N-1} \\
& +N(N-1) \frac{\rho^{2}}{N^{2}}\left(M_{s v}(r)\right)^{2}\left(1-\frac{\rho M_{v v}(r)}{N}\right)^{N-2} \\
& \underset{N \rightarrow \infty}{\longrightarrow}\left[\rho M_{s s}(r)+\rho^{2}\left(M_{s v}(r)\right)^{2}\right] \exp \left(-\rho M_{v v}(r)\right)
\end{aligned}
$$




\section{A.5.2 Tensorial two-point correlation functions}

Proceeding as in (68) and (69), the two-point correlation functions involving the orientation of the solid-fluid interface through $\boldsymbol{I}_{o}$ reduce to:

$$
\begin{aligned}
& \boldsymbol{F}_{o v}(\boldsymbol{r})=\rho \boldsymbol{M}_{o v}(\boldsymbol{r}) \exp \left(-\rho M_{v v}(r)\right) \\
& \boldsymbol{F}_{o s}(\boldsymbol{r})=\left[\rho \boldsymbol{M}_{o s}(\boldsymbol{r})+\rho^{2} \boldsymbol{M}_{o v}(\boldsymbol{r}) M_{v s}(r)\right] \exp \left(-\rho M_{v v}(r)\right) \\
& \boldsymbol{F}_{o o}(\boldsymbol{r})=\left[\rho \boldsymbol{M}_{o o}(\boldsymbol{r})+\rho^{2} \boldsymbol{M}_{o v}(\boldsymbol{r}) \cdot \boldsymbol{M}_{v o}(\boldsymbol{r})\right] \exp \left(-\rho M_{v v}(r)\right)
\end{aligned}
$$

where

$$
\begin{aligned}
& \boldsymbol{M}_{o v}(\boldsymbol{r})=\int \boldsymbol{O}(\boldsymbol{x}) V(\boldsymbol{x}+\boldsymbol{r}) \mathrm{d} V_{x} \\
& \boldsymbol{M}_{o s}(\boldsymbol{r})=\int \boldsymbol{O}(\boldsymbol{x}) S(\boldsymbol{x}+\boldsymbol{r}) \mathrm{d} V_{x} \\
& \boldsymbol{M}_{o o}(\boldsymbol{r})=\int \boldsymbol{O}(\boldsymbol{x}) \cdot \boldsymbol{O}(\boldsymbol{x}+\boldsymbol{r}) \mathrm{d} V_{x}
\end{aligned}
$$

These second order tensors possess the transverse isotropic symmetry w.r.t the direction $\boldsymbol{e}_{r}=\boldsymbol{r} / r$ and thus may be expressed in the basis $\left(\boldsymbol{e}_{r} \otimes \boldsymbol{e}_{r}, \mathbf{1}-\boldsymbol{e}_{r} \otimes \boldsymbol{e}_{r}\right)$ which is orthogonal for the dot product :

$$
\begin{aligned}
& \boldsymbol{M}_{o v}(\boldsymbol{r})=\frac{M_{s v}(r)}{3} \times \begin{cases}\left(\eta^{2}-\eta+1\right) \boldsymbol{e}_{r} \otimes \boldsymbol{e}_{r}-\frac{\eta^{2}-\eta-2}{2}\left(\mathbf{1}-\boldsymbol{e}_{r} \otimes \boldsymbol{e}_{r}\right) & \text { if } \eta \leq 1 \\
\mathbf{1} & \text { if } \eta>1\end{cases} \\
& \boldsymbol{M}_{o s}(\boldsymbol{r})=M_{s s}(r) \times\left(\eta^{2} \boldsymbol{e}_{r} \otimes \boldsymbol{e}_{r}+\frac{1-\eta^{2}}{2}\left(\mathbf{1}-\boldsymbol{e}_{r} \otimes \boldsymbol{e}_{r}\right)\right) \\
& \boldsymbol{M}_{o o}(\boldsymbol{r})=M_{s s}(r) \times\left(2 \eta^{2}-1\right)\left(\eta^{2} \boldsymbol{e}_{r} \otimes \boldsymbol{e}_{r}-\frac{1-\eta^{2}}{2}\left(\mathbf{1}-\boldsymbol{e}_{r} \otimes \boldsymbol{e}_{r}\right)\right)
\end{aligned}
$$

where the property (54) has been used whenever a product of two surface distributions is encountered. Note that only projections of the type $\boldsymbol{F}_{o i}(\boldsymbol{r})$ : $\left(\mathbf{1}+\boldsymbol{e}_{r} \otimes \boldsymbol{e}_{r}\right)$ for $i$ in $\{v, s, o\}$ are required for the evaluation of the two-point bounds on the permeability.

\section{A.6 Three-point correlation functions}

The three-point correlation functions defined in (53) can be derived for the Boolean model of spheres following the same steps as in $\S$ A.5:

$$
\begin{aligned}
F_{v v v}(\boldsymbol{r}, \boldsymbol{s}) & =\exp \left(-\rho M_{v v v}(\boldsymbol{r}, \boldsymbol{s})\right) \\
F_{v v s}(\boldsymbol{r}, \boldsymbol{s}) & =\rho M_{v v s}(\boldsymbol{r}, \boldsymbol{s}) \exp \left(-\rho M_{v v v}(\boldsymbol{r}, \boldsymbol{s})\right) \\
\boldsymbol{F}_{v v o}(\boldsymbol{r}, \boldsymbol{s}) & =\rho \boldsymbol{M}_{v v o}(\boldsymbol{r}, \boldsymbol{s}) \exp \left(-\rho M_{v v v}(\boldsymbol{r}, \boldsymbol{s})\right) \\
F_{v s s}(\boldsymbol{r}, \boldsymbol{s}) & =\left[\rho M_{v s s}(\boldsymbol{r}, \boldsymbol{s})+\rho^{2} M_{v s v}(\boldsymbol{r}, \boldsymbol{s}) M_{v v s}(\boldsymbol{r}, \boldsymbol{s})\right] \exp \left(-\rho M_{v v v}(\boldsymbol{r}, \boldsymbol{s})\right) \\
\boldsymbol{F}_{v s o}(\boldsymbol{r}, \boldsymbol{s}) & =\left[\rho \boldsymbol{M}_{v s o}(\boldsymbol{r}, \boldsymbol{s})+\rho^{2} M_{v s v}(\boldsymbol{r}, \boldsymbol{s}) \boldsymbol{M}_{v v o}(\boldsymbol{r}, \boldsymbol{s})\right] \exp \left(-\rho M_{v v v}(\boldsymbol{r}, \boldsymbol{s})\right) \\
\boldsymbol{F}_{v o o}(\boldsymbol{r}, \boldsymbol{s}) & =\left[\rho \boldsymbol{M}_{v o o}(\boldsymbol{r}, \boldsymbol{s})+\rho^{2} \boldsymbol{M}_{v o v}(\boldsymbol{r}, \boldsymbol{s}) \cdot \boldsymbol{M}_{v v o}(\boldsymbol{r}, \boldsymbol{s})\right] \exp \left(-\rho M_{v v v}(\boldsymbol{r}, \boldsymbol{s})\right)
\end{aligned}
$$


where the following geometrical quantities involving three spheres at the origin, $\boldsymbol{r}$ and $s$ appear:

$$
\begin{aligned}
M_{v v v}(\boldsymbol{r}, \boldsymbol{s}) & =\int 1-V(\boldsymbol{x}) V(\boldsymbol{x}+\boldsymbol{r}) V(\boldsymbol{x}+\boldsymbol{s}) \mathrm{d} V_{x} \\
M_{v v s}(\boldsymbol{r}, \boldsymbol{s}) & =\int V(\boldsymbol{x}) V(\boldsymbol{x}+\boldsymbol{r}) S(\boldsymbol{x}+\boldsymbol{s}) \mathrm{d} V_{x} \\
\boldsymbol{M}_{v v o}(\boldsymbol{r}, \boldsymbol{s}) & =\int V(\boldsymbol{x}) V(\boldsymbol{x}+\boldsymbol{r}) \boldsymbol{O}(\boldsymbol{x}+\boldsymbol{s}) \mathrm{d} V_{x} \\
M_{v s s}(\boldsymbol{r}, \boldsymbol{s}) & =\int V(\boldsymbol{x}) S(\boldsymbol{x}+\boldsymbol{r}) S(\boldsymbol{x}+\boldsymbol{s}) \mathrm{d} V_{x} \\
\boldsymbol{M}_{v s o}(\boldsymbol{r}, \boldsymbol{s}) & =\int V(\boldsymbol{x}) S(\boldsymbol{x}+\boldsymbol{r}) \boldsymbol{O}(\boldsymbol{x}+\boldsymbol{s}) \mathrm{d} V_{x} \\
\boldsymbol{M}_{v o o}(\boldsymbol{r}, \boldsymbol{s}) & =\int V(\boldsymbol{x}) \boldsymbol{O}(\boldsymbol{x}+\boldsymbol{r}) \cdot \boldsymbol{O}(\boldsymbol{x}+\boldsymbol{s}) \mathrm{d} V_{x}
\end{aligned}
$$

For example, $M_{v v v}(\boldsymbol{r}, \boldsymbol{s})$ is the volume of the union of three spheres of radius $R$ centred at $\mathbf{0}, \boldsymbol{r}$ and $\boldsymbol{s}$. Using the inclusion-exclusion formula, it is convenient to express the geometrical quantities (74) as functions of complementary geometrical quantities defined on the intersection of the three spheres:

$$
\begin{aligned}
M_{v v v}(\boldsymbol{r}, \boldsymbol{s}) & =\hat{M}_{v v v}(\boldsymbol{r}, \boldsymbol{s})+M_{v v}(r)+M_{v v}(s)+M_{v v}(t)-3 M_{v} \\
M_{v v s}(\boldsymbol{r}, \boldsymbol{s}) & =\hat{M}_{v v s}(\boldsymbol{r}, \boldsymbol{s})+M_{s v}(s)+M_{s v}(t)-M_{s} \\
\boldsymbol{M}_{v v o}(\boldsymbol{r}, \boldsymbol{s}) & =\hat{\boldsymbol{M}}_{v v o}(\boldsymbol{r}, \boldsymbol{s})+\boldsymbol{M}_{o v}(\boldsymbol{s})+\boldsymbol{M}_{o v}(\boldsymbol{t})-\boldsymbol{M}_{o} \\
M_{v s s}(\boldsymbol{r}, \boldsymbol{s}) & =-\hat{M}_{v s s}(\boldsymbol{r}, \boldsymbol{s})+M_{s s}(t)
\end{aligned}
$$

where $\boldsymbol{M}_{o}=\int \boldsymbol{O}(\boldsymbol{x}) \mathrm{d} V_{x}=\frac{4}{3} \pi R^{2} \mathbf{1}$ and

$$
\begin{aligned}
\hat{M}_{v v v}(\boldsymbol{r}, \boldsymbol{s}) & =\int \hat{V}(\boldsymbol{x}) \hat{V}(\boldsymbol{x}+\boldsymbol{r}) \hat{V}(\boldsymbol{x}+\boldsymbol{s}) \mathrm{d} V_{x} \\
\hat{M}_{v v s}(\boldsymbol{r}, \boldsymbol{s}) & =\int \hat{V}(\boldsymbol{x}) \hat{V}(\boldsymbol{x}+\boldsymbol{r}) S(\boldsymbol{x}+\boldsymbol{s}) \mathrm{d} V_{x} \quad \text { with } \hat{V}=1-V \\
\hat{\boldsymbol{M}}_{v v o}(\boldsymbol{r}, \boldsymbol{s}) & =\int \hat{V}(\boldsymbol{x}) \hat{V}(\boldsymbol{x}+\boldsymbol{r}) \boldsymbol{O}(\boldsymbol{x}+\boldsymbol{s}) \mathrm{d} V_{x} \\
\hat{M}_{v s s}(\boldsymbol{r}, \boldsymbol{s}) & =\int \hat{V}(\boldsymbol{x}) S(\boldsymbol{x}+\boldsymbol{r}) S(\boldsymbol{x}+\boldsymbol{s}) \mathrm{d} V_{x}
\end{aligned}
$$

For example, $\hat{M}_{v v v}(\boldsymbol{r}, \boldsymbol{s})$ is the volume of the intersection of three spheres. The expressions of the geometrical quantities in (74) and (76) involve several cases depending on the configuration of the three spheres (see Gibson and Scheraga, 1987). Let us denote

$$
a=\|\boldsymbol{r}\| / R \quad ; b=\|\boldsymbol{s}\| / R \quad ; \quad c=\|\boldsymbol{t}\| / R
$$


case 1

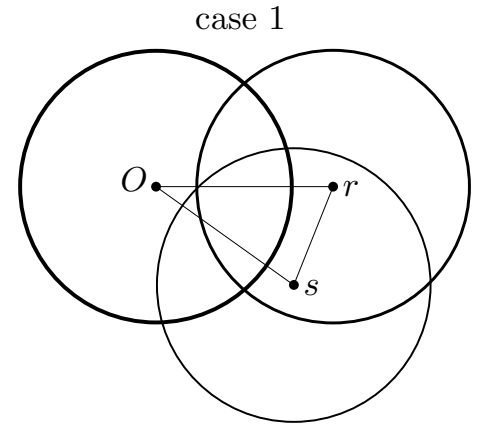

case 2

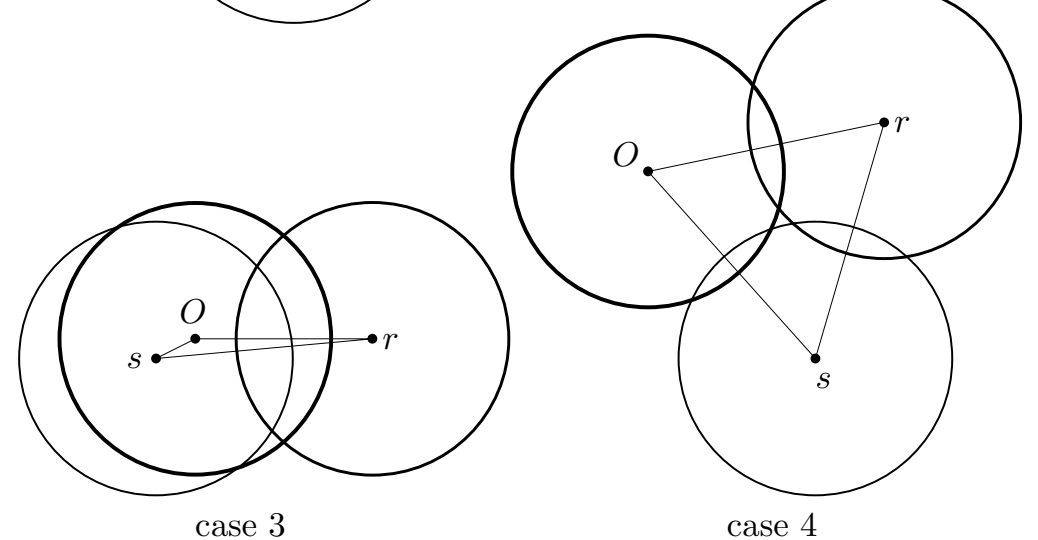

Figure 7: Configurations of three equal spheres, in the plane of their centres

the scaled distances between the sphere centres, so that the cosine of the angle between $\boldsymbol{r}$ and $\boldsymbol{s}$ is

$$
u=\boldsymbol{e}_{r} \cdot \boldsymbol{e}_{s}=\left(a^{2}+b^{2}-c^{2}\right) /(2 a b)
$$

Following Gibson and Scheraga (1987), let us introduce the quantities

$$
\begin{aligned}
q_{1} & =a\left(b^{2}+c^{2}-a^{2}\right) \quad ; \quad q_{2}=b\left(c^{2}+a^{2}-b^{2}\right) \quad ; \quad q_{3}=c\left(a^{2}+b^{2}-c^{2}\right) \\
w^{2} & =\left(a^{2}+b^{2}+c^{2}\right)^{2}-2\left(a^{4}+b^{4}+c^{4}\right)-a^{2} b^{2} c^{2}
\end{aligned}
$$

The triple intersection of the spheres is non-trivial (i.e. not empty or not equal to the intersection of two spheres) when $w^{2}>0$, which happens only if $a \leq 2$, $b \leq 2$ and $c_{-}<c<c_{+}$with

$$
c_{ \pm}=\frac{1}{2}\left[4\left(a^{2}+b^{2}\right)-2 a^{2} b^{2} \pm 2\left(16 a^{2} b^{2}-4 a^{2} b^{2}\left(a^{2}+b^{2}\right)+a^{4} b^{4}\right)^{1 / 2}\right]^{1 / 2}
$$

By symmetry, we present only the situations for which $b<a$, restricting to $r, s$ and $t$ smaller than $2 R$ :

- Case $1: 0<b<a<2$ and $c_{-}<c<c_{+}$

The triple intersection is non-trivial. From the formula of Gibson and 
Scheraga (1987) and derivation w.r.t. appropriate sphere radii one readily obtains (with the convention $0 \leq \tan ^{-1} \leq \pi$ ):

$$
\begin{aligned}
& \hat{M}_{v v v}(\boldsymbol{r}, \boldsymbol{s}) / R^{3}=\frac{w}{6}+\frac{4}{3}\left[\tan ^{-1} \frac{a w}{q_{1}}+\tan ^{-1} \frac{b w}{q_{2}}+\tan ^{-1} \frac{c w}{q_{3}}\right] \\
& -\left[a\left(1-\frac{a^{2}}{12}\right) \tan ^{-1} \frac{2 w}{q_{1}}+b\left(1-\frac{b^{2}}{12}\right) \tan ^{-1} \frac{2 w}{q_{2}}+c\left(1-\frac{c^{2}}{12}\right) \tan ^{-1} \frac{2 w}{q_{3}}\right] \\
& \hat{M}_{v v s}(\boldsymbol{r}, \boldsymbol{s}) / R^{2}=2 \tan ^{-1} \frac{c w}{q_{3}}+2 \tan ^{-1} \frac{b w}{q_{2}}-c \tan ^{-1} \frac{2 w}{q_{3}}-b \tan ^{-1} \frac{2 w}{q_{2}} \\
& \hat{M}_{v s s}(\boldsymbol{r}, \boldsymbol{s}) / R=\frac{2}{c} \tan ^{-1} \frac{2 w}{q_{3}}
\end{aligned}
$$

Further lengthy geometrical considerations lead to:

$$
\begin{array}{llll}
M_{v s s}=\frac{R}{c} \int_{\theta_{h}}^{\pi-\theta_{h}} \mathrm{~d} \theta=\frac{R}{c}\left(\pi-2 \theta_{h}\right) & \text { with } & \tan \left(\theta_{h}\right)=\frac{-u c}{\sqrt{4\left(1-u^{2}\right)-c^{2}}} \\
\boldsymbol{M}_{\text {vso }}=\frac{R}{c} \int_{\theta_{h}}^{\pi-\theta_{h}} \boldsymbol{n}_{s} \otimes \boldsymbol{n}_{s} \mathrm{~d} \theta & \text { with } & \boldsymbol{n}_{s}=\sqrt{1-\frac{c^{2}}{4}}\left(\cos \theta \boldsymbol{u}_{1}+\sin \theta \boldsymbol{u}_{2}\right)+\frac{c}{2} \boldsymbol{e}_{t} \\
\boldsymbol{M}_{\text {voo }}=R \frac{2-c^{2}}{2 c} \int_{\theta_{h}}^{\pi-\theta_{h}} \boldsymbol{n}_{r} \otimes \boldsymbol{n}_{s} \mathrm{~d} \theta & \text { with } & \boldsymbol{n}_{r}=\sqrt{1-\frac{c^{2}}{4}}\left(\cos \theta \boldsymbol{u}_{1}+\sin \theta \boldsymbol{u}_{2}\right)-\frac{c}{2} \boldsymbol{e}_{t}
\end{array}
$$

where $\left(\boldsymbol{u}_{1}, \boldsymbol{u}_{2}, \boldsymbol{e}_{t}\right)$ is a direct orthonormal basis defined by:

$$
\boldsymbol{u}_{1}=\frac{\boldsymbol{e}_{s} \wedge \boldsymbol{e}_{r}}{\sqrt{1-u^{2}}} \quad ; \quad \boldsymbol{u}_{2}=\frac{(b-a u) \boldsymbol{e}_{r}+(a-b u) \boldsymbol{e}_{s}}{c \sqrt{1-u^{2}}} \quad ; \quad \boldsymbol{e}_{t}=\frac{\boldsymbol{t}}{t}
$$

and

$$
\begin{aligned}
\hat{\boldsymbol{M}}_{v v o} & =\left(\hat{M}_{v v s}-b f\left(x_{b}\right)-c f\left(x_{c}\right)\right) \frac{\mathbf{1}}{3} \\
& +b f\left(x_{b}\right) \boldsymbol{e}_{s} \otimes \boldsymbol{e}_{s}+c f\left(x_{c}\right) \boldsymbol{e}_{t} \otimes \boldsymbol{e}_{t}-\frac{2}{3} h^{3}\left(\boldsymbol{u}_{2} \otimes \boldsymbol{e}_{t}+\frac{\boldsymbol{e}_{r}-u \boldsymbol{e}_{s}}{\sqrt{1-u^{2}}} \otimes \boldsymbol{e}_{s}\right)
\end{aligned}
$$

where

$$
\begin{gathered}
x_{b}=\frac{a-b u}{\sqrt{\left(1-u^{2}\right)\left(4-b^{2}\right)}} \quad ; \quad x_{c}=\frac{u c}{\sqrt{\left(1-u^{2}\right)\left(4-c^{2}\right)}} \\
h=R \sqrt{1-\frac{c^{2}}{4\left(1-u^{2}\right)}} \quad ; \quad f(x)=\frac{R}{2}\left[\pi / 2-\arcsin (x)-x \sqrt{1-x^{2}}\right]
\end{gathered}
$$

- Case 2 : $0<b<a$ and $a-b<c<c_{-}$

The triple intersection is included in the sphere centred at $s$ and equal to 
the intersection of the two spheres centred at $\mathbf{0}$ and $\boldsymbol{r}$.

$$
\begin{aligned}
M_{v v v}(\boldsymbol{r}, \boldsymbol{s}) & =M_{v v}(s)+M_{v v}(t)-M_{v} \\
M_{v v s}(\boldsymbol{r}, \boldsymbol{s}) & =M_{s v}(s)+M_{s v}(t)-M_{s} \\
\boldsymbol{M}_{v v o}(\boldsymbol{r}, \boldsymbol{s}) & =\boldsymbol{M}_{o v}(\boldsymbol{s})+\boldsymbol{M}_{o v}(\boldsymbol{t})-\boldsymbol{M}_{o} \\
M_{v s v}(\boldsymbol{r}, \boldsymbol{s}) & =M_{s v}(t) \quad ; \quad \boldsymbol{M}_{v o v}(\boldsymbol{r}, \boldsymbol{s})=\boldsymbol{M}_{o v}(\boldsymbol{t}) \\
M_{v s s}(\boldsymbol{r}, \boldsymbol{s}) & =M_{s s}(t) \quad ; \quad \boldsymbol{M}_{v o o}(\boldsymbol{r}, \boldsymbol{s})=\boldsymbol{M}_{o o}(\boldsymbol{t}) \\
\boldsymbol{M}_{v s o}(\boldsymbol{r}, \boldsymbol{s}) & =\boldsymbol{M}_{v o s}(\boldsymbol{r}, \boldsymbol{s})=\boldsymbol{M}_{o s}(\boldsymbol{t})
\end{aligned}
$$

- Case 3: $0<b<\sqrt{4-a^{2}}$ and $c_{+}<c<a+b$

The triple intersection is included in the sphere centred at $\mathbf{0}$ and equal to the intersection of the two spheres centred at $\boldsymbol{r}$ and $\boldsymbol{s}$.

$$
\begin{aligned}
& M_{v v v}(\boldsymbol{r}, \boldsymbol{s})=M_{v v}(r)+M_{v v}(s)-M_{v} \\
& M_{v v s}(\boldsymbol{r}, \boldsymbol{s})=M_{s v}(s) \quad ; \quad \boldsymbol{M}_{v v o}(\boldsymbol{r}, \boldsymbol{s})=\boldsymbol{M}_{o v}(\boldsymbol{s}) \\
& M_{v s v}(\boldsymbol{r}, \boldsymbol{s})=M_{s v}(r) \quad ; \quad \boldsymbol{M}_{v o v}(\boldsymbol{r}, \boldsymbol{s})=\boldsymbol{M}_{o v}(\boldsymbol{r}) \\
& M_{v s s}(\boldsymbol{r}, \boldsymbol{s})=\boldsymbol{M}_{v o o}(\boldsymbol{r}, \boldsymbol{s})=\boldsymbol{M}_{v s o}(\boldsymbol{r}, \boldsymbol{s})=\boldsymbol{M}_{v o s}(\boldsymbol{r}, \boldsymbol{s})=0
\end{aligned}
$$

- Case $4:\left(\sqrt{4-a^{2}}<b<a\right.$ and $\left.c_{+}<c<a+b\right)$ or $(0<b<2$ and $2<a<2+b)$

The triple intersection does not occur.

$$
\begin{aligned}
M_{v v v}(\boldsymbol{r}, \boldsymbol{s}) & =M_{v v}(r)+M_{v v}(s)+M_{v v}(t)-3 M_{v} \\
M_{v v s}(\boldsymbol{r}, \boldsymbol{s}) & =M_{s v}(s)+M_{s v}(t)-M_{s} ; \quad \boldsymbol{M}_{v v o}(\boldsymbol{r}, \boldsymbol{s})=\boldsymbol{M}_{o v}(\boldsymbol{s})+\boldsymbol{M}_{o v}(\boldsymbol{t})-\boldsymbol{M}_{o} \\
M_{v s v}(\boldsymbol{r}, \boldsymbol{s}) & =M_{s v}(r)+M_{s v}(t)-M_{s} ; \quad \boldsymbol{M}_{v o v}(\boldsymbol{r}, \boldsymbol{s})=\boldsymbol{M}_{o v}(\boldsymbol{r})+\boldsymbol{M}_{o v}(\boldsymbol{t})-\boldsymbol{M}_{o} \\
M_{v s s}(\boldsymbol{r}, \boldsymbol{s}) & =M_{s s}(t) ; \quad \boldsymbol{M}_{v o o}(\boldsymbol{r}, \boldsymbol{s})=\boldsymbol{M}_{o o}(\boldsymbol{t}) \\
\boldsymbol{M}_{v s o}(\boldsymbol{r}, \boldsymbol{s}) & =\boldsymbol{M}_{v o s}(\boldsymbol{r}, \boldsymbol{s})=\boldsymbol{M}_{o s}(\boldsymbol{t})
\end{aligned}
$$

\section{A.7 Alternative derivation of the scalar spatial correlation functions}

A powerful tool to derive the correlation functions involving scalar surface information such as $F_{v s}, F_{s s}, F_{v v s}$ or $F_{v s s}$ is to express $I_{s}$ as a function of $I_{v}$ :

$$
I_{s}(\boldsymbol{z}, R)=-\frac{\partial}{\partial R} I_{v}(\boldsymbol{z}, R)
$$

where the additional argument $R$ refers to the radius of the spheres used to build the Boolean model. Let us then introduce the functions:

$$
\begin{aligned}
F_{1}\left(R_{1}\right) & =\left\langle I_{v}\left(\boldsymbol{z}, R_{1}\right)\right\rangle=\exp \left(-\rho M_{1}\left(R_{1}\right)\right) \\
F_{2}\left(r, R_{1}, R_{2}\right) & =\left\langle I_{v}\left(\boldsymbol{z}, R_{1}\right) I_{v}\left(\boldsymbol{z}+\boldsymbol{r}, R_{2}\right)\right\rangle=\exp \left(-\rho M_{2}\left(r, R_{1}, R_{2}\right)\right) \\
F_{3}\left(\boldsymbol{r}, \boldsymbol{s}, R_{1}, R_{2}, R_{3}\right) & =\left\langle I_{v}\left(\boldsymbol{z}, R_{1}\right) I_{v}\left(\boldsymbol{z}+\boldsymbol{r}, R_{2}\right) I_{v}\left(\boldsymbol{z}+\boldsymbol{s}, R_{3}\right)\right\rangle=\exp \left(-\rho M_{3}\left(\boldsymbol{r}, \boldsymbol{s}, R_{1}, R_{2}, R_{3}\right)\right)
\end{aligned}
$$


where $M_{1}\left(R_{1}\right)=4 \pi R_{1}^{3} / 3$ is the volume of one ball of radius $R_{1} ; M_{2}\left(r, R_{1}, R_{2}\right)$ is the volume of the union of two balls of radii $R_{1}$ and $R_{2}$ whose centres are distant of $r$ :

$M_{2}\left(r, R_{1}, R_{2}\right)= \begin{cases}\frac{\pi}{12 r}\left(3\left(R_{1}-R_{2}\right)^{2}+2\left(R_{2}+R_{2}\right) r-r^{2}\right)\left(R_{1}+R_{2}+r\right)^{2} & \text { if } r \leq R_{1}+R_{2} \\ \frac{4 \pi}{3}\left(R_{1}^{3}+R_{2}^{3}\right) & \text { if } r>R_{1}+R_{2}\end{cases}$

and $M_{3}\left(\boldsymbol{r}, s, R_{1}, R_{2}, R_{3}\right)$ is the volume of the union of three balls of radii $R_{1}$, $R_{2}, R_{3}$ centred at $\mathbf{0}, \boldsymbol{r}$ and $\boldsymbol{s}$. The volume $M_{3}$ of the union of three spheres of unequal sizes can be deduced by the inclusion-exclusion formula from $M_{1}, M_{2}$ and the volume of the intersection of three spheres of unequal sizes provided in Gibson and Scheraga (1987).

By use of (86) in (53), derivation under the integrals in (87) with respect to $R_{1}, R_{2}$ or $R_{3}$ whenever appropriate yields:

$$
\begin{aligned}
F_{s} & =-\left.\frac{\partial}{\partial R_{1}} F_{1}\left(R_{1}\right)\right|_{R_{1}=R} \\
F_{v s} & =-\left.\frac{\partial}{\partial R_{2}} F_{2}\left(R_{1}, R_{2}\right)\right|_{R_{1}=R_{2}=R} \\
F_{s s} & =\left.\frac{\partial^{2}}{\partial R_{1} \partial R_{2}} F_{2}\left(R_{1}, R_{2}\right)\right|_{R_{1}=R_{2}=R} \\
F_{v v s} & =-\left.\frac{\partial}{\partial R_{3}} F_{3}\left(R_{1}, R_{2}, R_{3}\right)\right|_{R_{1}=R_{2}=R_{3}=R} \\
F_{v s s} & =\left.\frac{\partial^{2}}{\partial R_{2} \partial R_{3}} F_{3}\left(R_{1}, R_{2}, R_{3}\right)\right|_{R_{1}=R_{2}=R_{3}=R}
\end{aligned}
$$




\section{B Numerical integration}

The evaluation of the bounds of the permeability requires to compute integrals of the type:

$$
K_{i j, 2 \mathrm{pts}}=\frac{1}{6 \mu} \int_{r=0}^{\infty}\left(\mathbf{1}+\boldsymbol{e}_{r} \otimes \boldsymbol{e}_{r}\right):\left\langle\boldsymbol{A}_{i}(\boldsymbol{z}) \cdot \boldsymbol{A}_{j}(\boldsymbol{z}+\boldsymbol{r})\right\rangle r \mathrm{~d} r
$$

for the two-point bounds and:

$K_{i j, 3 \mathrm{pts}}=\frac{1}{4 \mu} \int_{r=0}^{\infty} \int_{s=0}^{\infty} \int_{u=-1}^{1} \boldsymbol{e}_{r} \cdot\left\langle I_{v}(\boldsymbol{z}) \boldsymbol{A}_{i}(\boldsymbol{z}+\boldsymbol{r}) \cdot \boldsymbol{A}_{j}(\boldsymbol{z}+\boldsymbol{s})\right\rangle \cdot \boldsymbol{e}_{s}\left(3 u^{2}-1\right) \mathrm{d} r \mathrm{~d} s \mathrm{~d} u$

for the three-point bounds, where $\boldsymbol{A}_{i}$ and $\boldsymbol{A}_{j}$ are linear combinations of the indicator functions (52), so that the terms in brackets are linear combinations of the spatial correlation functions (53).

For the Boolean model of spheres, the simple integrals of the type $K_{i j, 2 \mathrm{pts}}$ (90) for the two-point bounds have a vanishing integrand as soon as the two spheres do not intersect, that is for $r \geq 2 R$, and can easily be numerically integrated. The triple integrals of the type $K_{i j, 3 p t s}(91)$ have a vanishing integrand as soon as the three spheres do not intersect at all. However, the direct integration of $K_{i j, 3 p t s}$ as expressed in (91) has to be carried out on an infinite domain since the spheres centred at $\boldsymbol{r}$ and $\boldsymbol{s}$ may intersect for arbitrarily large values of $r$ and $s$.

To overcome this difficulty, in view of performing the numerical integration on finite domains, recall that for any admissible trial force field $\boldsymbol{f}$ :

$\boldsymbol{\nabla} \boldsymbol{P} \cdot \boldsymbol{K} \cdot \boldsymbol{\nabla} \boldsymbol{P} \leq \frac{2 \mu}{|\Omega|} \iiint_{\Omega} I_{v}(\boldsymbol{z})(\mathcal{G}(\boldsymbol{z}-\boldsymbol{x}) \cdot \boldsymbol{f}(\boldsymbol{x})):(\mathcal{G}(\boldsymbol{z}-\boldsymbol{y}) \cdot \boldsymbol{f}(\boldsymbol{y})) \mathrm{d} V_{x} \mathrm{~d} V_{y} \mathrm{~d} V_{z}$

In (92), one may rewrite the void indicator $I_{v}(\boldsymbol{z})$ as $\phi+\left(I_{v}(\boldsymbol{z})-\phi\right)$. The integral in the r.h.s. of (26) is then split in two terms: one for $\phi$ and one for $I_{v}(\boldsymbol{z})-\phi$. The former term is simply $\phi$ times the simpler bound:

$$
\frac{1}{|\Omega|} \iint_{\Omega} \boldsymbol{f}(\boldsymbol{z}) \cdot \boldsymbol{G}(\boldsymbol{z}-\boldsymbol{y}) \cdot \boldsymbol{f}(\boldsymbol{y}) \mathrm{d} V_{y} \mathrm{~d} V_{z}
$$

By following the subsequent steps of the companion article, it is a simple matter to show that $K_{i j, 3 p t s}$ can be decomposed as:

$$
K_{i j, 3 \mathrm{pts}}=\phi K_{i j, 2 \mathrm{pts}}+\delta K_{i j, 3 \mathrm{pts}}
$$

where

$\delta K_{i j, 3 \mathrm{pts}}=\frac{1}{4 \mu} \int_{r=0}^{\infty} \int_{s=0}^{\infty} \int_{u=-1}^{1} \boldsymbol{e}_{r} \cdot\left\langle\left(I_{v}(\boldsymbol{z})-\phi\right) \boldsymbol{A}_{i}(\boldsymbol{z}+\boldsymbol{r}) \cdot \boldsymbol{A}_{j}(\boldsymbol{z}+\boldsymbol{s})\right\rangle \cdot \boldsymbol{e}_{s}\left(3 u^{2}-1\right) \mathrm{d} r \mathrm{~d} s \mathrm{~d} u$ 
The term in brackets in $\delta K_{i j, 3 p t s}$ involves similar two- and three-point correla-

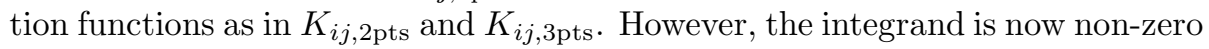
on a finite domain, which eases the numerical triple integration. The numerical integrations have been carried out using a Python interface to QUADPACK (Piessens et al, 1983). 